\title{
NEW WALL LAWS FOR THE UNSTEADY INCOMPRESSIBLE NAVIER-STOKES EQUATIONS ON ROUGH DOMAINS
}

\author{
Gabriel R. Barrenechea ${ }^{1,2}{ }^{\text {, Patrick Le Tallec }}{ }^{3}$ And Frédéric Valentin ${ }^{4}$
}

\begin{abstract}
Different effective boundary conditions or wall laws for unsteady incompressible NavierStokes equations over rough domains are derived in the laminar setting. First and second order unsteady wall laws are proposed using two scale asymptotic expansion techniques. The roughness elements are supposed to be periodic and the influence of the rough boundary is incorporated through constitutive constants. These constants are obtained by solving steady Stokes problems and so they are calculated only once. Numerical tests are presented to validate and compare the proposed boundary conditions.
\end{abstract}

Mathematics Subject Classification. 76M45, 76M10.

Received: November 8, 2001.

\section{INTRODUCTION}

Numerical simulation of flows over rough interfaces is a critical problem in CFD because of many situations involving rapidly varying micro structures near the wall. The direct solution of the Navier-Stokes equations in real 3D domains (with thousands of roughness elements in the computational domain) becomes a difficult task, specially when the interest is to simulate unsteady viscous flows. There are many practical problems where unsteady flows over rough boundaries are relevant such as:

- In aerodynamics, space shuttles are covered with tiles and its walls have an array of periodic gaps between the tiles. Similarly, in the drag control of an airplane wing, small injection jets are introduced over the wing in order to decrease the drag [4].

- In weather forecast the effects of hills, trees and buildings must be taken into account. In many climate applications, water waves should be included to properly simulate ocean-atmosphere interactions (see [15] and [12]).

- In optimal shape design, particularly in active control, the shapes are time dependent. In several cases it is possible to replace active shape control by a boundary control using wall laws or transpiration conditions [4].

\footnotetext{
Keywords and phrases. Wall law, unsteady Navier-Stokes equations, asymptotic analysis, rough boundary.

1 INRIA Projet M3N, Domaine de Voluceau, 78153 Le Chesnay, France. e-mail: Gabriel.Barrenechea@inria.fr

2 Departamento de Ingeniería Matemática, Universidad de Concepción, Casilla 160-C, Concepción, Chile.

3 École Polytechnique, 91128 Palaiseau Cedex, France. e-mail: Patrick.LeTallec@polytechnique.fr

${ }^{4}$ LNCC, Av. Getúlio Vargas, 333, 25651-070 Petrópolis - RJ, Brazil. e-mail: Valentin@lncc.br
} 
- In hemodynamics, the cell surfaces of the endothelial has the property to modify the wall shear stress produced by the flow field [22]. Therefore, wall laws could be useful in order to simulate in an accurate way the cell geometry influence on the blood flow.

The problem of fluid flow simulation over rough boundaries has already been studied mathematically in [9] where a domain decomposition method was proposed to construct wall laws for flows over periodic rough interfaces. This approach was extended to turbulent flows in [5] where some geometric dependent cases were considered. Their argument was analyzed in [1] for the Laplace equation and in [2] for the Stokes problem where the authors developed a theoretical framework coupled with a convergence analysis showing good performance of the boundary conditions.

Recently ( $c f .[3]$ ), effective boundary conditions (wall laws) for the steady-state incompressible Navier-Stokes equations on a rough domain with periodic roughness elements were derived within the framework of two-scale asymptotic expansion techniques (for a survey on such techniques, see [7] and [18]). The two-scale analysis of wall laws was pursued by considering steady laminar flow dominated by viscous effects in the roughness elements. Under this assumption, the flow near the wall tended to be Stokes-like with corrections due to convection. This choice led to the obtention of accurate numerical results as was pointed out in [3] and [21].

On the other hand, wall laws have been used for unsteady flows giving satisfactory numerical results (cf. [17] and [16]). However, the same wall laws have been used for both steady and unsteady flows without any mathematical justification. The purpose of this work is then to derive accurate, mathematically justified wall laws for time-dependent flows, assuming that the flow varies slowly, which implies that we have only one time scale to deal with. This choice has proven to be the right one, at least for the laminar flows we consider in this work. We introduce first and second order wall laws for unsteady flows. We observe that, for the first order approximation (and for the first order only) the same wall law is obtained for both steady [3] and unsteady flows, which gives a possible justification of the fact that the same wall laws are commonly used for both steady-state and unsteady flows. This is not the case for the second order approximation which gives us more accurate numerical results than the first order one as we shall show in the numerical results, where we validate the specific unsteady wall laws by several numerical simulations and we verify the approximation improvement produced by the second order wall law.

The outline of the paper is the following: Section 2 contains the description of the problem. First order approximation and the first order effective boundary condition are introduced in Section 3. Second order approximation and boundary conditions are derived in Section 4. Section 5 contains a brief description of the time discretization of our problem and the implementation of wall laws, and the validation is performed by numerical experiments in Section 6. Finally, some conclusions and perspectives of future work are given in Section 7 .

\section{Definition of the Problem}

We begin by describing a domain that is partially rough with periodic roughness elements. Let $\left(\mathbf{e}_{1}, \mathbf{e}_{2}\right)$ be an orthonormal basis of $\mathbb{R}^{2}$, and let $Y \subset \mathbb{R}^{2}$ be a semi-infinite domain in the $\mathbf{e}_{2}$ direction, such that the boundary of $Y$ is decomposed into three parts (Fig. 1):

$$
\partial Y=\partial Y_{1} \cup \partial Y_{2} \cup \partial Y_{3},
$$

where

$$
\partial Y_{1}=\{0\} \times\left[0, \infty\left[\quad, \quad \partial Y_{2}=\{2 \pi\} \times[0, \infty[,\right.\right.
$$

and $\partial Y_{3}$ is a connected Lipschitz bounded curve such that

$$
\partial Y_{1} \cap \partial Y_{3}=\{(0,0)\}, \partial Y_{2} \cap \partial Y_{3}=\{(2 \pi, 0)\} .
$$


Let $\varepsilon$ be a small positive real number, and let $Y^{\varepsilon}$ be the image of $Y$ by a dilatation of ratio $\varepsilon$ and center $(0,0)$. Further, let $\Theta^{\varepsilon}$ be the semi-infinite domain of $\mathbb{R}^{2}$ obtained by merging together all the images of $Y^{\varepsilon}$ by the translations by $2 \pi k \varepsilon \mathbf{e}_{1}$ where $k$ takes all the integer values. The infinite domain $\Theta^{\varepsilon}$ is contained in the half plane $x_{2} \geq 0$. Let $\Omega$ be a bounded domain of $\mathbb{R}^{2}$ intersecting the half plane $\left\{x_{2} \geq 0\right\}$. For simplicity, we suppose that this intersection is connected. Thus, for $\varepsilon$ small enough, $\Theta^{\varepsilon} \cap \Omega$ has a fast oscillating rough boundary, with wavelength of order $\varepsilon$ (Fig. 2). The amplitude of the roughness elements is also of order $\varepsilon$.

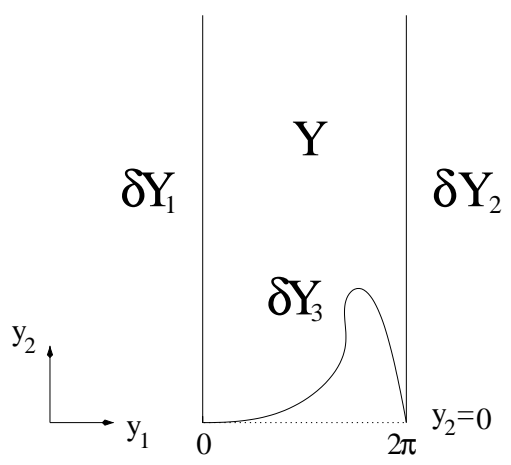

Figure 1. The cell $Y$.

We denote $\Omega^{\varepsilon}=\Theta^{\varepsilon} \cap \Omega$ and $\Gamma^{\varepsilon}$ the rough part of $\partial \Omega^{\varepsilon}$. We also denote by $\Omega^{\circ}=\left\{\mathbf{x} \in \Omega: x_{2}>0\right\}$ and $\Gamma^{\circ}=\partial \Omega^{\circ} \cap\left\{x_{2}=0\right\}$. When $\varepsilon \rightarrow 0, \overline{\Omega^{\varepsilon}}$ converges to $\overline{\Omega^{\circ}}$ in the sense of Hausdorff. As usual, we use notation $\left(x_{1}, x_{2}\right)$ and $\mathbf{y}:=\left(y_{1}, y_{2}\right)=\left(\frac{x_{1}}{\varepsilon}, \frac{x_{2}}{\varepsilon}\right)$ for the macroscopic and microscopic variables, respectively. We consider a unique time scale $t \in(0, T]$, where $T \in \mathbb{R}^{+}$is the final time of the process.

Let us introduce the space $L_{\mathrm{per}}^{2}(Y)$ of functions in $Y, 2 \pi$-periodic in the $y_{1}$ variable, and square integrable in $Y$, and the subspace $H_{\text {per }}^{1}(Y) \subset L_{\text {per }}^{2}(Y)$ of the functions whose first derivatives belong to $L_{\text {per }}^{2}(Y)$. We also introduce the following space

$$
\mathcal{S}_{\text {per }}(Y):=\left\{f \in L_{\text {per }}^{2}(Y) / \lim _{y_{2} \rightarrow \infty} f(\mathbf{y}) \mathrm{e}^{\alpha y_{2}}=0 \text { for some } \alpha>0\right\} .
$$

Without loss of generality we have chosen to work in two dimensions. Nonetheless, all that follows can be generalized to the three dimensional case.

We consider an unsteady fluid flow over a rough interface modeled by the usual unsteady incompressible Navier-Stokes equations

$$
\begin{aligned}
\frac{\partial \mathbf{u}^{\varepsilon}}{\partial t}+\mathbf{u}^{\varepsilon} \cdot \nabla \mathbf{u}^{\varepsilon}-\nu \Delta \mathbf{u}^{\varepsilon}+\nabla p^{\varepsilon} & =\mathbf{f} & & \text { in } \Omega^{\varepsilon} \times(0, T], \\
\nabla \cdot \mathbf{u}^{\varepsilon} & =0 & & \text { in } \Omega^{\varepsilon} \times(0, T], \\
\mathbf{u}^{\varepsilon} & =\mathbf{0} & & \text { on } \Gamma^{\varepsilon} \times(0, T], \\
\mathbf{u}^{\varepsilon} & =\mathbf{w} & & \text { in } \Omega^{\varepsilon} \text { at } t=0,
\end{aligned}
$$

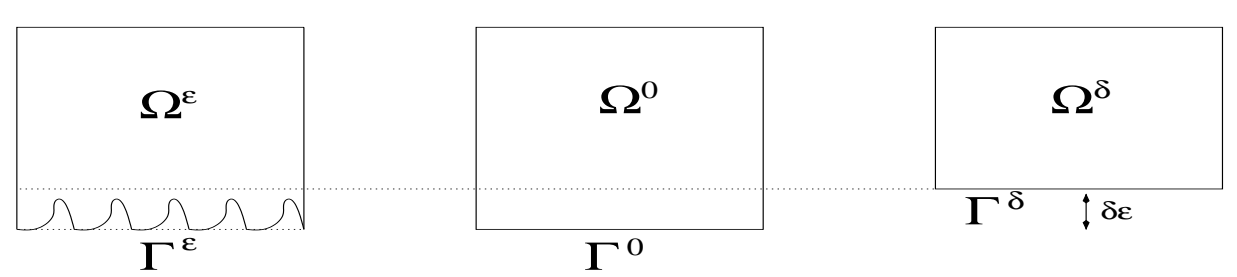

Figure 2. The domains $\Omega^{\varepsilon}, \Omega^{\circ}$ and $\Omega^{\delta}$. 
and for simplicity, we assume that the support of the source term $\mathbf{f}$ does not intersect $\Gamma^{\varepsilon}$. Of course, it is possible to assume other boundary conditions, typically, inflow-outflow boundary conditions, but they introduce only technical difficulties, and hence the present setting is sufficient for our derivation purposes. The initial velocity $\mathbf{w}$ is given and defined in $\Omega^{\circ}$.

The coefficient $\nu$ is the viscosity. When $\nu$ is small, the flow exhibits boundary layers near the walls. Thus, the problem has three characteristic lengths, namely, the macroscopic scale (linked to $\Omega^{\varepsilon}$ and $\mathbf{f}$ ), the Prandtl's boundary layer scale (of order $\sqrt{\nu}$ for laminar flows), and the roughness element scale $\varepsilon$. We are interested in the case where these scales are well separated, specially when $\sqrt{\nu} \gg \varepsilon$. Under this assumption, it is reasonable to expect a viscous sublayer of size $O(\varepsilon)$ due to the roughness elements inside the Prandtl's boundary layer. Thus, we set $\nu=\mu \varepsilon$, with $\mu$ being a real constant. This choice is convenient and permit to cover several practical applications, as numerically proved in [21]. Of course, other regimes with other asymptotic expansions are possible, but one has to keep in mind that asymptotic expansions are rather artificial since for practical applications, the viscosity and the geometry are both given and fixed. Therefore, adding this scaling law the problem can be rewritten as

$$
\begin{aligned}
\frac{\partial \mathbf{u}^{\varepsilon}}{\partial t}+\mathbf{u}^{\varepsilon} \cdot \nabla \mathbf{u}^{\varepsilon}-\mu \varepsilon \Delta \mathbf{u}^{\varepsilon}+\nabla p^{\varepsilon} & =\mathbf{f} & & \text { in } \Omega^{\varepsilon} \times(0, T], \\
\nabla \cdot \mathbf{u}^{\varepsilon} & =0 & & \text { in } \Omega^{\varepsilon} \times(0, T], \\
\mathbf{u}^{\varepsilon} & =\mathbf{0} & & \text { on } \Gamma^{\varepsilon} \times(0, T], \\
\mathbf{u}^{\varepsilon} & =\mathbf{w} & & \text { in } \Omega^{\varepsilon} \text { at } t=0 .
\end{aligned}
$$

We shall assume enough regularity on the data such that all the Navier-Stokes problems introduced below have isolated branches of solutions corresponding to laminar regimes [20].

In the following, we shall denote $\mathcal{L}^{\varepsilon}$ the partial differential operator

$$
\mathcal{L}^{\varepsilon}(\mathbf{u}, p)=\frac{\partial \mathbf{u}}{\partial t}+\mathbf{u} \cdot \nabla \mathbf{u}-\mu \varepsilon \Delta \mathbf{u}+\nabla p
$$

and make the important assumption that the mean flow is not strongly affected by the roughness elements, i.e., the solution of (2.2) is a perturbation of the solution of the following problem:

$$
\begin{aligned}
\frac{\partial \mathbf{u}^{\circ}}{\partial t}+\mathbf{u}^{\circ} \cdot \nabla \mathbf{u}^{\circ}-\mu \varepsilon \Delta \mathbf{u}^{\circ}+\nabla p^{\circ} & =\mathbf{f} & & \text { in } \Omega^{\circ} \times(0, T], \\
\nabla \cdot \mathbf{u}^{\circ} & =0 & & \text { in } \Omega^{\circ} \times(0, T], \\
\mathbf{u}^{\circ} & =\mathbf{0} & & \text { on } \Gamma^{\circ} \times(0, T], \\
\mathbf{u}^{\circ} & =\mathbf{w} & & \text { in } \Omega^{\circ} \text { at } t=0 .
\end{aligned}
$$

In the case of the linear steady state Stokes equations, the above assumption can in fact be rigorously proved (see [2]). We also assume that the solution of the above system describes a laminar flow, in such a way that we have the following Prandtl's length scales on $\Gamma^{\circ}$ :

$$
\begin{gathered}
\frac{\partial u_{1}^{\circ}}{\partial x_{2}}=O\left(\nu^{-1 / 2}\right), \frac{\partial^{2} u_{1}^{\circ}}{\partial x_{2}^{2}}=O\left(\nu^{-1}\right), \frac{\partial^{2} u_{1}^{\circ}}{\partial x_{1} \partial x_{2}}=O\left(\nu^{-1 / 2}\right), \\
\frac{\partial u_{1}^{\circ}}{\partial t}=O(1), \frac{\partial^{2} u_{1}^{\circ}}{\partial x_{2} \partial t}=O\left(\nu^{-1}\right) .
\end{gathered}
$$


We finally recall some derivation rules. For a function $\phi(\mathbf{x})=\tilde{\phi}(\mathbf{x}, \mathbf{y})$ we have

$$
\begin{aligned}
\nabla \phi & =\frac{1}{\varepsilon} \nabla_{\mathbf{y}} \tilde{\phi}+\nabla \tilde{\phi} \\
\nabla \cdot \phi & =\frac{1}{\varepsilon} \nabla_{\mathbf{y}} \cdot \tilde{\phi}+\nabla \cdot \tilde{\phi} \\
\Delta \phi & =\frac{1}{\varepsilon^{2}} \Delta_{\mathbf{y}} \tilde{\phi}+\frac{2}{\varepsilon} \Delta_{\mathbf{x y}} \tilde{\phi}+\Delta \tilde{\phi}
\end{aligned}
$$

where $\Delta_{\mathbf{x y}} \tilde{\phi}:=\Sigma_{i} \frac{\partial}{\partial x_{i}}\left(\frac{\partial \tilde{\phi}}{\partial y_{i}}\right)$, and the absence of subindexes denotes derivation with respect to $\mathbf{x}$.

As we shall see in the numerical tests, zeroth order approximation (2.3) fails to correctly predict the velocity and pressure inside the boundary layer, as these variables are influenced by the roughness elements. Therefore, we are interested in constructing higher order approximation problems based on asymptotic expansion techniques.

\section{First order approximation}

\subsection{The ansatz}

At the first stage of the calculation, we want to correct the error generated by the fact that we have replaced $\mathbf{u}^{\varepsilon}$ by $\mathbf{u}^{\circ}$ in $(2.2)$. Since $\Omega^{\varepsilon} \subset \Omega^{\circ}$, the error arises from the fact that $\mathbf{u}^{\circ}$ does not satisfy the no-slip boundary conditions on $\Gamma^{\varepsilon}$. However, since $\mathbf{u}^{\circ}$ vanishes on $\Gamma^{\circ}$ and since $\Gamma^{\varepsilon}$ is close to $\Gamma^{\circ}$, the error is small and given by the following Taylor expansion in the $x_{2}$ variable:

$$
\mathbf{u}^{\circ}(\mathbf{x}, t)=-\varepsilon \frac{\partial \mathbf{u}^{\circ}}{\partial \mathbf{n}}\left(x_{1}, 0, t\right) \frac{x_{2}}{\varepsilon}+\varepsilon^{2} \frac{\partial^{2} \mathbf{u}^{\circ}}{\partial \mathbf{n}^{2}}\left(x_{1}, 0, t\right)\left(\xi(\mathbf{x}) \frac{x_{2}}{\varepsilon}\right)^{2},
$$

for all $(\mathbf{x}, t) \in \Gamma^{\varepsilon} \times(0, T]$ and where $0<\xi<1$. Here, the assumption that $(2.2)$ describes a laminar flow implies that, at leading order,

$$
\mathbf{u}^{\circ}(\mathbf{x}, t) \approx-\varepsilon \frac{\partial \mathbf{u}^{\circ}}{\partial \mathbf{n}}\left(x_{1}, 0, t\right) \frac{x_{2}}{\varepsilon}
$$

Now, since $u_{1}^{\circ}=0$ on $\Gamma^{\circ} \times(0, T]$, we have $\frac{\partial u_{1}^{\circ}}{\partial \mathbf{s}}=0$ on $\Gamma^{\circ} \times(0, T]$, and using a Taylor series, Prandtl's scales and the incompressibility condition we arrive at

$$
\frac{\partial u_{1}^{\circ}}{\partial \mathbf{s}}=-\frac{\partial u_{2}^{\circ}}{\partial \mathbf{n}}=O(\sqrt{\varepsilon})
$$

inside the boundary layer. Thus, using (3.1), we arrive at the following expression for the error

$$
\mathbf{u}^{\circ}(\mathbf{x}, t)=-\varepsilon \frac{\partial u_{1}^{\circ}}{\partial \mathbf{n}}\left(x_{1}, 0, t\right) \frac{x_{2}}{\varepsilon} \mathbf{e}_{1} \quad \forall(\mathbf{x}, t) \in \Gamma^{\varepsilon} \times(0, T]
$$

Now, we observe that from the Prandtl scales we have that the error is of $O(\sqrt{\varepsilon})$ magnitude. Hence, we propose the following ansatz

$$
\begin{aligned}
& \mathbf{u}^{\varepsilon}(\mathbf{x}, t) \approx \mathbf{u}^{\circ}(\mathbf{x}, t)+\sqrt{\varepsilon} \mathbf{u}_{B L}^{1}(\mathbf{x}, \mathbf{y}, t) \\
& p^{\varepsilon}(\mathbf{x}, t) \approx p^{\circ}(\mathbf{x}, t)+\sqrt{\varepsilon} p_{B L}^{1}(\mathbf{x}, \mathbf{y}, t),
\end{aligned}
$$


where the terms $\mathbf{u}_{B L}^{1}(\mathbf{x}, \mathbf{y}, t)$ and $p_{B L}^{1}(\mathbf{x}, \mathbf{y}, t)$ are called boundary layer correctors because they correct at first order the fast oscillating error when replacing $\mathbf{u}^{\varepsilon}$ by $\mathbf{u}^{\circ}$ in (2.2), and they are of $O(1)$ magnitude. The influence of the correctors is mainly restricted to the boundary layer. The term (3.2) is the error at leading order which is expressed in terms of the product between a function of the macroscopic variable and a fast oscillating periodic term. Therefore, a natural way to look for correctors is

$$
\begin{aligned}
& \mathbf{u}_{B L}^{1}(\mathbf{x}, \mathbf{y}, t)=\sqrt{\varepsilon} \frac{\partial u_{1}^{\circ}}{\partial \mathbf{n}}\left(x_{1}, 0, t\right)\left(\chi^{1}(\mathbf{y})-\overline{\chi^{1}}\right) \\
& p_{B L}^{1}(\mathbf{x}, \mathbf{y}, t)=\sqrt{\varepsilon} \frac{\partial u_{1}^{\circ}}{\partial \mathbf{n}}\left(x_{1}, 0, t\right) \pi^{1}(\mathbf{y})
\end{aligned}
$$

where $\chi^{1}$ (resp. $\left.\pi^{1}\right)$ is a function with values in $\mathbb{R}^{2}$, (resp. $\mathbb{R}$ ), periodic in the $y_{1}$ direction, and $\overline{\chi^{1}} \in \mathbb{R}^{2}$ such that $\chi^{1}-\overline{\chi^{1}}$ and $\pi^{1}$ decay exponentially fast to zero as $y_{2}$ tends to infinity (see Th. 3.1 below). We may note that, even if the correction depends on $\mathbf{x}$ and $t$, the one time scale assumption leads us to the fact that the dependence on $t$ is only present in the slow variable; this will have a direct impact in the calculation of the homogenization constant below.

Now, the leading order of $\mathcal{L}^{\varepsilon}\left(\mathbf{u}^{\circ}+\sqrt{\varepsilon} \mathbf{u}_{B L}^{1}, p^{\circ}+\sqrt{\varepsilon} p_{B L}^{1}\right)-\mathbf{f}$ is

$$
\mathcal{L}^{\varepsilon}\left(\mathbf{u}^{\circ}+\sqrt{\varepsilon} \mathbf{u}_{B L}^{1}, p^{\circ}+\sqrt{\varepsilon} p_{B L}^{1}\right)-\mathbf{f} \approx \frac{\partial u_{1}^{\circ}}{\partial \mathbf{n}}\left(x_{1}, 0, t\right)\left(-\mu \Delta_{\mathbf{y}} \chi^{1}+\nabla_{\mathbf{y}} \pi^{1}\right) .
$$

At a first glance, the convective term $\frac{\partial u_{1}^{\circ}}{\partial \mathbf{n}}\left(x_{1}, 0, t\right) \mathbf{u}^{\circ} \cdot \nabla_{\mathbf{y}} \chi^{1}$ looks like of the same order, but since $\nabla_{\mathbf{y}} \chi^{1}$ decays to zero exponentially fast as $y_{2}$ goes to infinity and $\mathbf{u}^{\circ}$ vanishes on $\Gamma^{\circ}$, this term is actually smaller. Likewise,

$$
\begin{aligned}
\nabla \cdot\left(\mathbf{u}^{\circ}+\sqrt{\varepsilon} \mathbf{u}_{B L}^{1}\right) & \approx \frac{\partial u_{1}^{\circ}}{\partial \mathbf{n}}\left(x_{1}, 0, t\right) \nabla_{\mathbf{y}} \cdot \chi^{1}+\varepsilon \frac{\partial^{2} u_{1}^{\circ}}{\partial \mathbf{n} \partial x_{1}}\left(x_{1}, 0, t\right) \chi_{1}^{1} \\
& \approx \frac{\partial u_{1}^{\circ}}{\partial \mathbf{n}}\left(x_{1}, 0, t\right) \nabla_{\mathbf{y}} \cdot \chi^{1} .
\end{aligned}
$$

Therefore, in order to correct these errors, correctors $\chi^{1}, \pi^{1}$ and the constant vector $\overline{\chi^{1}}$ should satisfy:

$$
\begin{array}{rlr}
-\mu \Delta_{\mathbf{y}} \chi^{1}+\nabla_{\mathbf{y}} \pi^{1} & =\mathbf{0} & \text { in } Y, \\
\nabla_{\mathbf{y}} \cdot \chi^{1} & =0 & \text { in } Y, \\
\chi^{1}-\overline{\chi^{1}} & =y_{2} \mathbf{e}_{1} \quad \text { on } \partial Y_{3}, \\
\chi^{1}-\overline{\chi^{1}} \in \mathcal{S}_{\mathrm{per}}(Y)^{2} & , \quad \pi^{1} \in \mathcal{S}_{\mathrm{per}}(Y) .
\end{array}
$$

Now, it is not difficult to realize that, in general, this corrector problem does not have a solution. Indeed, it suffices to take $\partial Y_{3}=\left\{y_{2}=1\right\}$, and in that case, the solution $\chi^{1}-\overline{\chi^{1}}=(1,0)$ does not tend to zero. That is why we relax the definition of this problem, and we only ask the triplet $\left(\chi^{1}, \overline{\chi^{1}}, \pi^{1}\right)$ to satisfy

$$
\begin{array}{rlrl}
-\mu \Delta_{\mathbf{y}} \chi^{1}+\nabla_{\mathbf{y}} \pi^{1} & =\mathbf{0} & \text { in } Y, \\
\nabla_{\mathbf{y}} \cdot \chi^{1} & =0 & 0 & \text { in } Y, \\
\chi^{1} & =y_{2} \mathbf{e}_{1} & \text { on } \partial Y_{3}, \\
\chi^{1}-\overline{\chi^{1}} \in \mathcal{S}_{\mathrm{per}}(Y)^{2} & , & \pi^{1} \in \mathcal{S}_{\mathrm{per}}(Y) .
\end{array}
$$

This cell problem is well posed, as stated in the following result, whose proof can be found in [3].

Theorem 3.1. There exists a unique pair of functions $\left(\chi^{1}, \pi^{1}\right)$ and a unique vector $\overline{\chi^{1}} \in \mathbb{R}^{2}$ such that $\chi^{1}-\overline{\chi^{1}} \in$ $H_{\mathrm{per}}^{1}(Y)^{2} \cap \mathcal{S}_{\mathrm{per}}(Y)^{2}, \pi^{1} \in L_{\mathrm{per}}^{2}(Y) \cap \mathcal{S}_{\mathrm{per}}(Y)$, and (3.7) is satisfied in a weak sense. Moreover, $\chi^{1}$ is an horizontal 
vector, i.e., $\overline{\chi^{1}}=\overline{\chi_{1}^{1}} \mathbf{e}_{1}$, and the constant $\overline{\chi_{1}^{1}}$ is bounded as follows

$$
0 \leq \overline{\chi_{1}^{1}} \leq H_{\max }:=\max \left\{y_{2}:\left(y_{1}, y_{2}\right) \in \partial Y_{3}\right\}
$$

Remark 3.1. We observe that even for an unsteady problem one has to solve just a steady Stokes problem in the cell $Y$, and hence the homogenization constant $\overline{\chi^{1}}$ is calculated only once at the beginning of the calculation. On the other hand, even for periodic roughness elements, here we allow general non-periodic assumption for the correctors. In other words, we do not impose the periodicity in the macroscopic scales, which was a common fact in the first works concerning wall laws ( $c f .[9])$.

Now, from the definition of the correctors (3.4) we have that

$$
\mathbf{u}^{\circ}+\sqrt{\varepsilon} \mathbf{u}_{B L}^{1}=-\varepsilon \overline{\chi^{1}} \frac{\partial u_{1}^{\circ}}{\partial \mathbf{n}}\left(x_{1}, 0, t\right) \quad \text { on } \Gamma^{\varepsilon} \times(0, T],
$$

which shows that adding $\sqrt{\varepsilon} \mathbf{u}_{B L}^{1}$ to $\mathbf{u}^{\circ}$ does not improve the error since it remains of the same order. However, a closer inspection shows that now, the error is no longer fast oscillating. Therefore, it can be corrected by modifying the ansatz in the following way:

$$
\begin{aligned}
& \mathbf{u}^{\varepsilon}(\mathbf{x}, t) \approx \mathbf{u}^{1}(\mathbf{x}, t)+\sqrt{\varepsilon} \mathbf{u}_{B L}^{1}(\mathbf{x}, \mathbf{y}, t), \\
& p^{\varepsilon}(\mathbf{x}, t) \approx p^{1}(\mathbf{x}, t)+\sqrt{\varepsilon} p_{B L}^{1}(\mathbf{x}, \mathbf{y}, t),
\end{aligned}
$$

where the pair $\left(\mathbf{u}^{1}, p^{1}\right)$ is a solution of the following macroscopic problem: find $\left(\mathbf{u}^{1}, p^{1}\right)$ such that

$$
\begin{aligned}
\frac{\partial \mathbf{u}^{1}}{\partial t}+\mathbf{u}^{1} \cdot \nabla \mathbf{u}^{1}-\mu \varepsilon \Delta \mathbf{u}^{1}+\nabla p^{1} & =\mathbf{f} & & \text { in } \Omega^{\circ} \times(0, T], \\
\nabla \cdot \mathbf{u}^{1} & =0 & & \text { in } \Omega^{\circ} \times(0, T], \\
\mathbf{u}^{1} & =\mathbf{0} & & \text { on } \partial \Omega^{\circ} \backslash \Gamma^{\circ} \times(0, T], \\
u_{1}^{1} & =\varepsilon \overline{\chi_{1}^{1}} \frac{\partial u_{1}^{\circ}}{\partial \mathbf{n}} & & \text { on } \Gamma^{\circ} \times(0, T], \\
u_{2}^{1} & =0 & & \text { on } \Gamma^{\circ} \times(0, T], \\
\mathbf{u}^{1} & =\mathbf{w} & & \text { in } \Omega^{\circ} \text { at } t=0 .
\end{aligned}
$$

\subsection{The related effective boundary condition}

In practice, the computation of $\left(\mathbf{u}^{\circ}, p^{\circ}\right)$ and $\left(\mathbf{u}^{1}, p^{1}\right)$ require to solve two Navier-Stokes problems in $\Omega^{\circ}$. Alternatively one may notice that near $\Gamma^{\circ}, \mathbf{u}^{1} \approx \mathbf{u}^{\circ}$, which shows that the boundary condition on $\Gamma^{\circ} \times(0, T]$ can be replaced by the Navier boundary condition

$$
u_{1}^{1}=\varepsilon \overline{\chi_{1}^{1}} \frac{\partial u_{1}^{1}}{\partial \mathbf{n}} \quad \text { on } \Gamma^{\circ} \times(0, T] .
$$

Using the same argument, we modify the definition of $\mathbf{u}_{B L}^{1}$ and $p_{B L}^{1}$ using $\mathbf{u}^{1}$ instead of $\mathbf{u}^{\circ}$. We remark that (3.1) is still valid for $\mathbf{u}^{1}$, using now first order wall law (3.10) instead of the no-slip condition on $\Gamma^{\circ} \times(0, T]$. However, (3.9) may be ill-posed since the constant $\overline{\chi_{1}^{1}}$ is positive (cf. Th. 3.1) and the variational formulation contains the term

$$
-\frac{\mu}{\overline{\chi_{1}^{1}}} \int_{\Gamma^{\circ}} u_{1}^{1} v_{1} .
$$

To avoid this difficulty, we introduce the domain $\Omega^{\delta}$ (see Fig. 2):

$$
\Omega^{\delta}=\Omega^{\circ} \cap\left\{x_{2}>\delta \varepsilon\right\}
$$


and the (fictitious) boundary $\Gamma^{\delta}=\partial \Omega^{\delta} \cap\left\{x_{2}=\delta \varepsilon\right\}$, and we shall solve (3.9) in $\Omega^{\delta}$ rather than in $\Omega^{\circ}$.

The following Taylor expansion on $u_{1}^{1}$

$$
\begin{aligned}
u_{1}^{1}\left(x_{1}, 0, t\right) & =u_{1}^{1}\left(x_{1}, \delta \varepsilon, t\right)+\delta \varepsilon \frac{\partial u_{1}^{1}}{\partial \mathbf{n}}\left(x_{1}, \delta \varepsilon, t\right)+\frac{\delta^{2} \varepsilon^{2}}{2} \frac{\partial^{2} u_{1}^{1}}{\partial \mathbf{n}^{2}}\left(x_{1}, \theta \delta \varepsilon, t\right), \\
\frac{\partial u_{1}^{1}}{\partial \mathbf{n}}\left(x_{1}, 0, t\right) & =\frac{\partial u_{1}^{1}}{\partial \mathbf{n}}\left(x_{1}, \delta \varepsilon, t\right)+\delta \varepsilon \frac{\partial^{2} u_{1}^{1}}{\partial \mathbf{n}^{2}}\left(x_{1}, \theta^{\prime} \delta \varepsilon, t\right),
\end{aligned}
$$

with $0<\theta, \theta^{\prime}<1$, gives the first order effective boundary conditions

$$
\begin{aligned}
\mu \varepsilon \frac{\partial u_{1}^{1}}{\partial \mathbf{n}}-\frac{\mu}{\overline{\chi_{1}^{1}}-\delta} u_{1}^{1} & =0, \\
u_{2}^{1} & =0,
\end{aligned}
$$

on $\Gamma^{\delta} \times(0, T]$.

Remark 3.2. Despite considering the problem to be unsteady, first order wall law (3.12) is exactly the same as the one obtained for the steady problem $[3,21]$. This provides a good explanation for the fact that classical steady wall laws have being used for unsteady flows with reasonable success [17]. We shall see that this is not the case for second order wall laws.

Remark 3.3. It is easily seen that the effective first order condition is equivalent (at this order of approximation) to a no-slip condition on a flat wall at an average height, computed by the asymptotic expansion, which is known in fluid mechanics as the mean effective height (see [10]). This shall not be the case in general for the second order effective condition.

\section{THE SECOND ORDER APPROXIMATION}

\subsection{The ansatz}

In order to improve the approximation of $\mathbf{u}^{\varepsilon}$ and $p^{\varepsilon}$, we propose the ansatz

$$
\begin{aligned}
\mathbf{u}^{\varepsilon} & \approx \mathbf{u}^{1}+\sqrt{\varepsilon} \mathbf{u}_{B L}^{1}(\mathbf{x}, \mathbf{y}, t)+\varepsilon \mathbf{u}_{B L}^{2}(\mathbf{x}, \mathbf{y}, t), \\
p^{\varepsilon} & \approx p^{1}+\sqrt{\varepsilon} p_{B L}^{1}(\mathbf{x}, \mathbf{y}, t)+\varepsilon p_{B L}^{2}(\mathbf{x}, \mathbf{y}, t),
\end{aligned}
$$

where the first order boundary layer terms have already been computed. Let us evaluate the error made when we substitute $\left(\mathbf{u}^{1}+\sqrt{\varepsilon} \mathbf{u}_{B L}^{1}, p^{1}+\sqrt{\varepsilon} p_{B L}^{1}\right)$ in (2.2). This error is, at the leading order, given by

$$
\mathcal{L}^{\varepsilon}\left(\mathbf{u}^{1}+\sqrt{\varepsilon} \mathbf{u}_{B L}^{1}, p^{1}+\sqrt{\varepsilon} p_{B L}^{1}\right)-\mathbf{f}=\frac{1}{\sqrt{\varepsilon}} \mathbf{u}^{1} \cdot \nabla_{\mathbf{y}} \mathbf{u}_{B L}^{1}+\sqrt{\varepsilon} \mathbf{u}_{B L}^{1} \cdot \nabla \mathbf{u}^{1}+\mathbf{u}_{B L}^{1} \cdot \nabla_{\mathbf{y}} \mathbf{u}_{B L}^{1}+\sqrt{\varepsilon} \frac{\partial \mathbf{u}_{B L}^{1}}{\partial t} .
$$

Now, to simplify the calculations, we give a more explicit expression for each term above. To do this, we use the definition of $\mathbf{u}_{B L}^{1},(3.1)$, the first order wall law, $\overline{\chi^{1}}=\overline{\chi_{1}^{1}} \mathbf{e}_{1}$, and Taylor series and we get:

- $\frac{1}{\sqrt{\varepsilon}} \mathbf{u}^{1} \cdot \nabla_{\mathbf{y}} \mathbf{u}_{B L}^{1} \approx \varepsilon\left(\frac{\partial u_{1}^{1}}{\partial \mathbf{n}}\right)^{2}\left(x_{1}, 0, t\right)\left(\overline{\chi^{1}}-y_{2} \mathbf{e}_{1}\right) \cdot \nabla_{\mathbf{y}} \chi^{1}$,

- $\sqrt{\varepsilon}\left(\mathbf{u}_{B L}^{1} \cdot \nabla\right) \mathbf{u}^{1} \approx-\varepsilon\left(\frac{\partial u_{1}^{1}}{\partial \mathbf{n}}\right)^{2}\left(x_{1}, 0, t\right) \chi_{2}^{1} \mathbf{e}_{1}$,

- $\left(\mathbf{u}_{B L}^{1} \cdot \nabla_{\mathbf{y}}\right) \mathbf{u}_{B L}^{1} \approx \varepsilon \nabla_{\mathbf{y}} \chi^{1} \cdot\left(\chi^{1}-\overline{\chi^{1}}\right)\left(\frac{\partial u_{1}^{1}}{\partial \mathbf{n}}\right)^{2}\left(x_{1}, 0, t\right)$,

- $\sqrt{\varepsilon} \frac{\partial \mathbf{u}_{B L}^{1}}{\partial t}=\varepsilon\left(\chi^{1}-\overline{\chi^{1}}\right) \frac{\partial^{2} u_{1}^{1}}{\partial \mathbf{n} \partial t}$. 
Therefore, the leading order term of $\mathcal{L}^{\varepsilon}\left(\mathbf{u}^{1}+\sqrt{\varepsilon} \mathbf{u}_{B L}^{1}, p^{1}+\sqrt{\varepsilon} p_{B L}^{1}\right)-\mathbf{f}$ is

$$
\begin{array}{r}
\mathcal{L}^{\varepsilon}\left(\mathbf{u}^{1}+\sqrt{\varepsilon} \mathbf{u}_{B L}^{1}, p^{1}+\sqrt{\varepsilon} p_{B L}^{1}\right)-\mathbf{f} \approx \varepsilon\left(\left(\chi^{1}(\mathbf{y})-y_{2} \mathbf{e}_{1}\right) \cdot \nabla_{\mathbf{y}} \chi^{1}(\mathbf{y})-\chi_{2}^{1}(\mathbf{y}) \mathbf{e}_{1}\right)\left(\frac{\partial u_{1}^{1}}{\partial \mathbf{n}}\right)^{2}\left(x_{1}, 0, t\right) \\
+\varepsilon\left(\chi^{1}(\mathbf{y})-\overline{\chi^{1}}\right) \frac{\partial^{2} u_{1}^{1}}{\partial \mathbf{n} \partial t}\left(x_{1}, 0, t\right)
\end{array}
$$

which is of $O(1)$ order of magnitude.

On the other hand, the error on the divergence is

$$
\nabla \cdot\left(\mathbf{u}^{1}+\sqrt{\varepsilon} \mathbf{u}_{B L}^{1}\right) \approx \varepsilon \frac{\partial^{2} u_{1}^{1}}{\partial \mathbf{n} \partial x_{1}}\left(x_{1}, 0, t\right)\left(\chi^{1}-\overline{\chi^{1}}\right) \cdot \mathbf{e}_{1}
$$

but, since $\left|\varepsilon \frac{\partial^{2} u_{1}^{1}}{\partial \mathbf{n} \partial x_{1}}\left(x_{1}, 0, t\right)\right|=O(\sqrt{\varepsilon})$, this error does not need to be corrected at leading order.

The error on the boundary condition is:

$$
\left(\mathbf{u}^{1}+\sqrt{\varepsilon} \mathbf{u}_{B L}^{1}\right) \approx \frac{x_{2}^{2}}{2 \varepsilon^{2}} \frac{\partial^{2} u_{1}^{1}}{\partial \mathbf{n}^{2}}\left(x_{1}, 0, t\right) \varepsilon^{2} \mathbf{e}_{1} \quad \text { on } \Gamma^{\varepsilon} \times(0, T]
$$

As in Section 3, we notice that the errors are given by products of fast oscillating periodic terms by slowly varying functions, involving three dominating terms, namely $\frac{\partial^{2} u_{1}^{1}}{\partial \mathbf{n}^{2}}\left(x_{1}, 0, t\right), \frac{\partial^{2} u_{1}^{1}}{\partial \mathbf{n} \partial t}\left(x_{1}, 0, t\right)$, and $\left(\frac{\partial u_{1}^{1}}{\partial \mathbf{n}}\right)^{2}\left(x_{1}, 0, t\right)$. Therefore, to both have correctors of $O(1)$ magnitude and correct this error, it is natural to look for correctors of the form:

$$
\begin{aligned}
\mathbf{u}_{B L}^{2}(\mathbf{x}, \mathbf{y}, t)= & \varepsilon\left(\chi^{2}(\mathbf{y})-\overline{\chi^{2}}\right)\left(\frac{\partial u_{1}^{1}}{\partial \mathbf{n}}\right)^{2}\left(x_{1}, 0, t\right)+\varepsilon\left(\chi^{3}(\mathbf{y})-\overline{\chi^{3}}\right) \frac{\partial^{2} u_{1}^{1}}{\partial \mathbf{n}^{2}}\left(x_{1}, 0, t\right) \\
& +\varepsilon\left(\chi^{4}(\mathbf{y})-\overline{\chi^{4}}\right) \frac{\partial^{2} u_{1}^{1}}{\partial \mathbf{n} \partial t}\left(x_{1}, 0, t\right), \\
p_{B L}^{2}(\mathbf{x}, \mathbf{y}, t)= & \varepsilon \pi^{2}(\mathbf{y})\left(\frac{\partial u_{1}^{1}}{\partial \mathbf{n}}\right)^{2}\left(x_{1}, 0, t\right)+\varepsilon \pi^{3}(\mathbf{y}) \frac{\partial^{2} u_{1}^{1}}{\partial \mathbf{n}^{2}}\left(x_{1}, 0, t\right) \\
& +\varepsilon \pi^{4}(\mathbf{y}) \frac{\partial^{2} u_{1}^{1}}{\partial \mathbf{n} \partial t}\left(x_{1}, 0, t\right),
\end{aligned}
$$

where again $\chi^{2}, \chi^{3}, \chi^{4}$ (resp. $\pi^{2}, \pi^{3}, \pi^{4}$ ) are functions taking values in $\mathbb{R}^{2}$, (resp. $\mathbb{R}$ ) and periodic in the $y_{1}$ direction, $\overline{\chi^{2}}, \overline{\chi^{3}}, \frac{\chi}{\chi^{4}} \in \mathbb{R}^{2}$ and we assume that $\chi^{2}-\overline{\chi^{2}}, \chi^{3}-\overline{\chi^{3}}, \chi^{4}-\overline{\chi^{4}}, \pi^{2}, \pi^{3}$ and $\pi^{4}$ decay to zero exponentially fast as $y_{2}$ goes to infinity. To correct the error, from $(4.2)$, the triplet $\left(\chi^{2}, \overline{\chi^{2}}, \pi^{2}\right)$ must satisfy:

$$
\begin{aligned}
-\mu \Delta_{\mathbf{y}} \chi^{2}+\nabla_{\mathbf{y}} \pi^{2} & =-\left(\left(\chi^{1}-y_{2} \mathbf{e}_{1}\right) \cdot \nabla_{\mathbf{y}} \chi^{1}-\chi_{2}^{1} \mathbf{e}_{1}\right) & & \text { in } Y \\
\nabla_{\mathbf{y}} \cdot \chi^{2} & =0 & & \text { in } Y, \\
\chi^{2} & =\mathbf{0} & & \text { on } \partial Y_{3}, \\
\chi^{2}-\overline{\chi^{2}} & \in \mathcal{S}_{\mathrm{per}}(Y)^{2}, \pi^{2} \in \mathcal{S}_{\mathrm{per}}(Y), & &
\end{aligned}
$$


whereas the triplet $\left(\chi^{3}, \overline{\chi^{3}}, \pi^{3}\right)$ satisfies

$$
\begin{array}{rlrl}
-\mu \Delta_{\mathbf{y}} \chi^{3}+\nabla_{\mathbf{y}} \pi^{3} & =\mathbf{0} & & \text { in } Y, \\
\nabla_{\mathbf{y}} \cdot \chi^{3} & =0 & & \text { in } Y, \\
\chi^{3} & =-\frac{y_{2}^{2}}{2} \mathbf{e}_{1} & & \text { on } \partial Y_{3}, \\
\chi^{3}-\overline{\chi^{3}} \in \mathcal{S}_{\mathrm{per}}(Y)^{2} & , \quad \pi^{3} \in \mathcal{S}_{\mathrm{per}}(Y), &
\end{array}
$$

and the triplet $\left(\chi^{4}, \overline{\chi^{4}}, \pi^{4}\right)$ satisfies

$$
\begin{aligned}
-\mu \Delta_{\mathbf{y}} \chi^{4}+\nabla_{\mathbf{y}} \pi^{4} & =-\left(\chi^{1}-\overline{\chi^{1}}\right) & & \text { in } Y, \\
\nabla_{\mathbf{y}} \cdot \chi^{4} & =0 & & \text { in } Y, \\
\chi^{4} & =\mathbf{0} & & \text { on } \partial Y_{3}, \\
\chi^{4}-\overline{\chi^{4}} \in \mathcal{S}_{\text {per }}(Y)^{2} & , \pi^{4} \in \mathcal{S}_{\text {per }}(Y) . & &
\end{aligned}
$$

Remark 4.1. These local (cell) problems were derived by doing the same considerations made in Section 3 regarding the boundary conditions on $\partial Y_{3}$. On the other hand, the new corrector $\left(\chi^{4}, \pi^{4}\right)$, and the homogenization constant $\overline{\chi^{4}}$, are associated with the time derivative term and therefore they were not present in the steady second order approximation [3].

Theorem 4.1. There exist unique pairs $\left(\chi^{2}, \pi^{2}\right),\left(\chi^{3}, \pi^{3}\right)$ and $\left(\chi^{4}, \pi^{4}\right)$ and unique constant vectors $\overline{\chi^{2}}, \overline{\chi^{3}}$ and $\overline{\chi^{4}}$ such that $\chi^{i}-\overline{\chi^{i}} \in H^{1}(Y)^{2} \cap \mathcal{S}_{\mathrm{per}}(Y)^{2}, \pi^{i} \in \mathcal{S}_{\mathrm{per}}(Y), i=2,3,4$, weak solutions of (4.4), (4.5) and (4.6), respectively.

Proof. The proof of the result for Problem (4.5) is completely analogous to the proof of Theorem 3.1 (see [3] for the details). The existence result for Problems (4.4) and (4.6) is detailed in Appendix A.

Remark 4.2. For the new cell problems introduced to treat the second order, we also note that by integrating by parts the divergence free conditions on $\chi^{2}, \chi^{3}$ and $\chi^{4}$, we obtain that

$$
\overline{\chi_{2}^{2}}=0, \overline{\chi_{2}^{3}}=0, \overline{\chi_{2}^{4}}=0
$$

At this stage we observe once again that, with the definition (4.3) of the correctors, the error on the boundary condition is not corrected, but it is not dependent on the fast variable neither. Hence, as we did in previous section, we redefine our ansatz as

$$
\begin{aligned}
& \mathbf{u}^{\varepsilon} \approx \mathbf{u}^{2}+\sqrt{\varepsilon} \mathbf{u}_{B L}^{1}(\mathbf{x}, \mathbf{y}, t)+\varepsilon \mathbf{u}_{B L}^{2}(\mathbf{x}, \mathbf{y}, t) \\
& p^{\varepsilon} \approx p^{2}+\sqrt{\varepsilon} p_{B L}^{1}(\mathbf{x}, \mathbf{y}, t)+\varepsilon p_{B L}^{2}(\mathbf{x}, \mathbf{y}, t),
\end{aligned}
$$


where we look for second order macroscopic approximations of $\left(\mathbf{u}^{\varepsilon}, p^{\varepsilon}\right)$ which are solutions of the following boundary value problem: find $\left(\mathbf{u}^{2}, p^{2}\right)$ such that

$$
\begin{array}{rlrl}
\frac{\partial \mathbf{u}^{2}}{\partial t}+\mathbf{u}^{2} \cdot \nabla \mathbf{u}^{2}-\mu \varepsilon \Delta \mathbf{u}^{2}+\nabla p^{2}= & \mathbf{f} & & \text { in } \Omega^{\circ} \times(0, T], \\
\nabla \cdot \mathbf{u}^{2}= & 0 \quad & \text { in } \Omega^{\circ} \times(0, T], \\
\mathbf{u}^{2}= & \mathbf{0} \quad & \text { on } \partial \Omega^{\circ} \backslash \Gamma^{\circ} \times(0, T], \\
u_{1}^{2}= & \overline{\chi_{1}^{1}} \frac{\partial u_{1}^{1}}{\partial \mathbf{n}}+\varepsilon^{2}\left(\overline{\chi_{1}^{2}}\left(\frac{\partial u_{1}^{1}}{\partial \mathbf{n}}\right)^{2}+\overline{\chi_{1}^{3}} \frac{\partial^{2} u_{1}^{1}}{\partial \mathbf{n}^{2}}\right. \\
& +\overline{\chi_{1}^{4}} \frac{\partial^{2} u_{1}^{1}}{\partial \mathbf{n} \partial t} \quad \text { on } \Gamma^{\circ} \times(0, T], \\
u_{2}^{2}= & 0 \quad \text { on } \Gamma^{\circ} \times(0, T], \\
\mathbf{u}^{2}= & \mathbf{w} \quad \text { in } \Omega^{\circ} \text { at } t=0 .
\end{array}
$$

\subsection{The related effective boundary condition}

As in Section 3.2, it is more convenient to compute $\left(\mathbf{u}^{2}, p^{2}\right)$ by changing the boundary conditions on $\Gamma^{\circ}$ slightly. Indeed, if $\overline{\chi_{1}^{1}} \neq 0$ one may use the second order effective boundary conditions

$$
\begin{aligned}
\mu \varepsilon \frac{\partial u_{1}^{2}}{\partial \mathbf{n}}-\frac{\mu}{\overline{\chi_{1}^{1}}} u_{1}^{2}+\frac{\mu}{\overline{\chi_{1}^{1}}} \varepsilon^{2}\left(\overline{\chi_{1}^{2}}\left(\frac{\partial u_{1}^{2}}{\partial \mathbf{n}}\right)^{2}+\overline{\chi_{1}^{3}} \frac{\partial^{2} u_{1}^{2}}{\partial \mathbf{n}^{2}}+\overline{\chi_{1}^{4}} \frac{\partial^{2} u_{1}^{2}}{\partial \mathbf{n} \partial t}\right) & =0, \\
u_{2}^{2} & =0
\end{aligned}
$$

on $\Gamma^{\circ} \times(0, T]$.

As for the first order approximation, the boundary value problem in $\Omega^{\circ}$ may be ill-posed. However, it is possible to construct a (possibly) well-posed problem in $\Omega^{\delta}$. Using a Taylor series for every term above, and neglecting all terms of $O(\varepsilon)$ we obtain the following wall laws in $\Gamma^{\delta} \times(0, T]$ :

$$
\begin{aligned}
\mu \varepsilon \frac{\partial u_{1}^{2}}{\partial \mathbf{n}}-\frac{\mu}{\overline{\chi_{1}^{1}}-\delta} u_{1}^{2}+\varepsilon^{2} \frac{\mu}{\overline{\chi_{1}^{1}}-\delta}\left(\left(\delta \overline{\chi_{1}^{1}}-\frac{\delta^{2}}{2}+\overline{\chi_{1}^{3}}\right) \frac{\partial^{2} u_{1}^{2}}{\partial \mathbf{n}^{2}}+\overline{\chi_{1}^{2}}\left(\frac{\partial u_{1}^{2}}{\partial \mathbf{n}}\right)^{2}+\overline{\chi_{1}^{4}} \frac{\partial^{2} u_{1}^{2}}{\partial \mathbf{n} \partial t}\right) & =0, \\
u_{2}^{2} & =0,
\end{aligned}
$$

on $\Gamma^{\delta} \times(0, T]$.

Now, these boundary conditions are rather complicated to implement due to the presence of second derivatives and squares of derivatives. That is why we transform them into boundary conditions which involve only first derivatives, and which also include pressure gradients and convective effects near the boundary (which have been shown to be important $(c f .[17]))$. First, by using the first order approximation of $\frac{\partial u_{1}^{2}}{\partial \mathbf{n}}$ given by (3.12) we get

$$
\varepsilon^{2} \frac{\mu}{\left(\overline{\chi_{1}^{1}}-\delta\right)} \overline{\chi_{1}^{2}}\left(\frac{\partial u_{1}^{2}}{\partial \mathbf{n}}\right)^{2} \approx \frac{\overline{\chi_{1}^{2}} \mu}{\left(\overline{\chi_{1}^{1}}-\delta\right)^{3}}\left(u_{1}^{2}\right)^{2},
$$

and

$$
\varepsilon^{2} \frac{\mu}{\left(\overline{\chi_{1}^{1}}-\delta\right)} \overline{\chi_{1}^{4}} \frac{\partial^{2} u_{1}^{2}}{\partial \mathbf{n} \partial t} \approx \varepsilon \frac{\mu}{\left(\overline{\chi_{1}^{1}}-\delta\right)^{2}} \overline{\chi_{1}^{4}} \frac{\partial u_{1}^{2}}{\partial t}
$$


Further, the Navier-Stokes equations restricted to the boundary $\Gamma^{\delta}$ indicate that at leading order we have

$$
\mu \varepsilon \frac{\partial^{2} u_{1}^{2}}{\partial \mathbf{n}^{2}}\left(x_{1}, \delta \varepsilon, t\right) \approx \frac{\partial p^{2}}{\partial x_{1}}\left(x_{1}, \delta \varepsilon, t\right)+\frac{\partial u_{1}^{2}}{\partial t}\left(x_{1}, \delta \varepsilon, t\right) .
$$

Therefore, from above considerations we obtain the following set of boundary conditions on $\Gamma^{\delta}$ :

$$
\begin{aligned}
\mu \varepsilon \frac{\partial u_{1}^{2}}{\partial \mathbf{n}}-\frac{\mu}{\overline{\chi_{1}^{1}}-\delta} u_{1}^{2}+\frac{1}{\overline{\chi_{1}^{1}}-\delta}\left(\varepsilon\left(\delta \overline{\chi_{1}^{1}}-\frac{\delta^{2}}{2}+\overline{\chi_{1}^{3}}\right) \frac{\partial p^{2}}{\partial x_{1}}\right. \\
\left.+\varepsilon\left(\delta \overline{\chi_{1}^{1}}-\frac{\delta^{2}}{2}+\overline{\chi_{1}^{3}}+\mu \frac{\overline{\chi_{1}^{4}}}{\left.\overline{\left(\chi_{1}^{1}\right.}-\delta\right)}\right) \frac{\partial u_{1}^{2}}{\partial t}+\mu \frac{\overline{\chi_{1}^{2}}}{\left.\overline{\left(\chi_{1}^{1}\right.}-\delta\right)^{2}}\left(u_{1}^{2}\right)^{2}\right)=0, \\
u_{2}^{2}=0,
\end{aligned}
$$

on $\Gamma^{\delta} \times(0, T]$.

Remark 4.3. The wall law (4.13) is different to (4.9), but the approximation error associated with each boundary law is smaller than the actual leading error.

\subsection{Summary: The proposed effective problems}

The first order initial boundary value problem in $\Omega^{\delta} \times(0, T]$ is the following:

$$
\begin{aligned}
\frac{\partial \mathbf{u}^{1}}{\partial t}+\mathbf{u}^{1} \cdot \nabla \mathbf{u}^{1}-\mu \varepsilon \Delta \mathbf{u}^{1}+\nabla p^{1} & =\mathbf{f} & & \text { in } \Omega^{\delta} \times(0, T], \\
\nabla \cdot \mathbf{u}^{1} & =0 & & \text { in } \Omega^{\delta} \times(0, T], \\
\mathbf{u}^{1} & =\mathbf{0} & & \text { on } \partial \Omega^{\delta} \backslash \Gamma^{\delta} \times(0, T], \\
\mu \varepsilon \frac{\partial u_{1}^{1}}{\partial \mathbf{n}}-\frac{\mu}{\overline{\chi_{1}^{1}}-\delta} u_{1}^{1} & =0 & & \text { on } \Gamma^{\delta} \times(0, T], \\
u_{2}^{1} & =0 & & \text { on } \Gamma^{\delta} \times(0, T], \\
\mathbf{u}^{1} & =\mathbf{w} & & \text { in } \Omega^{\delta} \text { at } t=0,
\end{aligned}
$$

where $\overline{\chi_{1}^{1}}$ is calculated from $(3.7)$.

The second order initial boundary value problem in $\Omega^{\delta} \times(0, T]$ is:

$$
\begin{aligned}
\frac{\partial \mathbf{u}^{2}}{\partial t}+\mathbf{u}^{2} . \nabla \mathbf{u}^{2}-\mu \varepsilon \Delta \mathbf{u}^{2}+\nabla p^{2} & =\mathbf{f} & & \text { in } \Omega^{\delta} \times(0, T], \\
\nabla \cdot \mathbf{u}^{2} & =0 & & \text { in } \Omega^{\delta} \times(0, T], \\
\mathbf{u}^{2} & =0 & & \text { on } \partial \Omega^{\delta} \backslash \Gamma^{\delta} \times(0, T], \\
\mu \varepsilon \frac{\partial u_{1}^{2}}{\partial \mathbf{n}}+C_{1} u_{1}^{2}+C_{2}^{\varepsilon} \frac{\partial p^{2}}{\partial x_{1}}+C_{3}^{\varepsilon} \frac{\partial u_{1}^{2}}{\partial t}+C_{4}\left(u_{1}^{2}\right)^{2} & =0 & & \text { on } \Gamma^{\delta} \times(0, T], \\
u_{2}^{2} & =0 & & \text { on } \Gamma^{\delta} \times(0, T], \\
\mathbf{u}^{2} & =\mathbf{w} & & \text { at } t=0,
\end{aligned}
$$


where

$$
\begin{aligned}
C_{1} & =-\frac{\mu}{\overline{\chi_{1}^{1}}-\delta} \\
C_{2}^{\varepsilon} & =\frac{\varepsilon}{\overline{\chi_{1}^{1}}-\delta}\left(\delta \overline{\chi_{1}^{1}}-\frac{\delta^{2}}{2}+\overline{\chi_{1}^{3}}\right) \\
C_{3}^{\varepsilon} & =\frac{\varepsilon}{\overline{\chi_{1}^{1}}-\delta}\left(\delta \overline{\chi_{1}^{1}}-\frac{\delta^{2}}{2}+\overline{\chi_{1}^{3}}+\mu \frac{\overline{\chi_{1}^{4}}}{\left.\overline{\left(\overline{\chi_{1}^{1}}\right.}-\delta\right)}\right) \\
C_{4} & =\mu \frac{\overline{\chi_{1}^{2}}}{\overline{\left(\overline{\chi_{1}^{1}}-\delta\right)^{3}}}
\end{aligned}
$$

and where $\overline{\chi^{2}}, \overline{\chi^{3}}$ and $\overline{\chi^{4}}$ are calculated from (4.4), (4.5) and (4.6) respectively.

For $\delta$ large enough, (4.14) and (4.15) may have a solution. We shall not discuss that subject in this paper, but we shall solve (4.14) and (4.15) numerically in Section 6, and compare its solution with the original flow.

\section{Numerical AND implementational aspects}

The numerical validation of the procedure proposed herein is performed using a 2D unsteady incompressible Navier-Stokes code implementing the Operator Sppliting scheme originally proposed in [11], in a linear version proposed in [19], also known as the fractional step $\theta$-scheme. In order to describe the method, let $\Delta t$ be a given time step, let us denote $t^{n}=n \Delta t$ and $\mathbf{f}^{n}:=\mathbf{f}\left(x, t^{n}\right)$. The precise formulation of the method is as follows: Let $\theta \in\left(0, \frac{1}{3}\right), \alpha, \beta \in(0,1)$, such that $\alpha+\beta=1$, hence, if $\mathbf{u}_{0}$ is given and divergence free in $\Omega^{\delta}$, compute $\mathbf{u}^{1}, \mathbf{u}^{2}, \ldots$ via

$$
\begin{aligned}
\frac{\mathbf{u}^{n+\theta}-\mathbf{u}^{n}}{\theta \Delta t}-\alpha \nu \Delta \mathbf{u}^{n+\theta}+\nabla p^{n+\theta} & =\alpha \mathbf{f}^{n+\theta}+\beta \mathbf{f}^{n}+\beta \nu \Delta \mathbf{u}^{n}-\mathbf{u}^{n} \cdot \nabla \mathbf{u}^{n} \quad \text { in } \Omega^{\delta} \\
\nabla \cdot \mathbf{u}^{n+\theta} & =0 \quad \text { in } \Omega^{\delta} \\
\mathbf{u}^{n+\theta} & =\mathbf{0} \quad \text { on } \partial \Omega^{\delta} \backslash \Gamma^{\delta}
\end{aligned}
$$

$$
\begin{aligned}
& \frac{\mathbf{u}^{n+1-\theta}-\mathbf{u}^{n+\theta}}{(1-2 \theta) \Delta t}-\beta \nu \Delta \mathbf{u}^{n+1-\theta}+\mathbf{u}^{*} \cdot \nabla \mathbf{u}^{n+1-\theta}=\beta \mathbf{f}^{n+1-\theta}+\alpha \mathbf{f}^{n+\theta}+\alpha \nu \Delta \mathbf{u}^{n+\theta}-\nabla p^{n+\theta} \quad \text { in } \Omega^{\delta}, \\
& \mathbf{u}^{n+1-\theta}=\mathbf{0} \quad \text { on } \partial \Omega^{\delta} \backslash \Gamma^{\delta} \\
& \frac{\mathbf{u}^{n+1}-\mathbf{u}^{n+1-\theta}}{\theta \Delta t}-\alpha \nu \Delta \mathbf{u}^{n+1}+\nabla p^{n+1}=\alpha \mathbf{f}^{n+1}+\beta \mathbf{f}^{n+1-\theta}+\beta \nu \Delta \mathbf{u}^{n+1-\theta}-\mathbf{u}^{*} \cdot \nabla \mathbf{u}^{n+1-\theta} \quad \text { in } \Omega^{\delta} \\
& \nabla \cdot \mathbf{u}^{n+1}=0 \quad \text { in } \Omega^{\delta} \\
& \mathbf{u}^{n+1}=\mathbf{0} \quad \text { on } \partial \Omega^{\delta} \backslash \Gamma^{\delta},
\end{aligned}
$$

where

$$
\begin{gathered}
\theta=1-\frac{1}{\sqrt{2}}, \\
\alpha=\frac{1-2 \theta}{1-\theta} \quad, \quad \beta=\frac{\theta}{1-\theta}, \\
\mathbf{u}^{*}=\frac{2 \theta-1}{\theta} \mathbf{u}^{n}+\frac{1-\theta}{\theta} \mathbf{u}^{n+\theta} .
\end{gathered}
$$


Now, we describe the implementation of our wall laws in the framework of this method. First order wall law reads

$$
\begin{aligned}
& u_{1}=C_{1} \frac{\partial u_{1}}{\partial \mathbf{n}} \\
& u_{2}=0
\end{aligned}
$$

on $\Gamma^{\delta} \times(0, T]$, where $C_{1}$ is a negative constant. Inspiring us in [11], this law is implemented in the following way:

$$
\alpha \frac{\partial u_{1}^{n+\theta}}{\partial \mathbf{n}}-\frac{\alpha}{C_{1}} u_{1}^{n+\theta}=-\beta \frac{\partial u_{1}^{n}}{\partial \mathbf{n}}+\frac{\beta}{C_{1}} u_{1}^{n},
$$

and $u_{2}^{n+\theta}=0$ on $\Gamma^{\delta}$, for the first Stokes-like problem (5.1),

$$
\beta \frac{\partial u_{1}^{n+1-\theta}}{\partial \mathbf{n}}-\frac{\beta}{C_{1}} u_{1}^{n+1-\theta}=-\alpha \frac{\partial u_{1}^{n+\theta}}{\partial \mathbf{n}}+\frac{\alpha}{C_{1}} u_{1}^{n+\theta},
$$

and $u_{2}^{n+1-\theta}=0$ on $\Gamma^{\delta}$, for the advection-diffusion-reaction problem (5.2), and

$$
\alpha \frac{\partial u_{1}^{n+1}}{\partial \mathbf{n}}-\frac{\alpha}{C_{1}} u_{1}^{n+1}=-\beta \frac{\partial u_{1}^{n+1-\theta}}{\partial \mathbf{n}}+\frac{\beta}{C_{1}} u_{1}^{n+1-\theta},
$$

and $u_{2}^{n+1}=0$ on $\Gamma^{\delta}$, for the second Stokes-like problem (5.3). This implementation has been proved to be second order accurate in time (see [6]).

In an analogous way, second order wall law imposes also $u_{2}=0$ on $\Gamma^{\delta} \times(0, T]$, which allows us to forget this variable and concentrate us only on $u_{1}$. Denoting $u_{1}$ simply by $u$, second order wall law reads

$$
C_{1} \frac{\partial u}{\partial t}+\frac{\partial u}{\partial \mathbf{n}}+C_{2} \frac{\partial p}{\partial \mathbf{s}}+C_{3} u^{2}-C_{4} u=0,
$$

on $\Gamma^{\delta} \times(0, T]$, where $C_{1}, C_{2}, C_{3}, C_{4}$ are real constants.

We remark now that since second order wall law is a nonlinear boundary condition which also contains pressure gradients, we have to propose a linearization and an operator sppliting inside this boundary conditions. Combining [11] and [19] we propose the following implementation of this law

$C_{1} \frac{u^{n+\theta}-u^{n}}{\theta \Delta t}+\alpha \frac{\partial u^{n+\theta}}{\partial \mathbf{n}}+C_{2} \frac{\partial p^{n+\theta}}{\partial \mathbf{s}}-\alpha C_{4} u^{n+\theta}=-\beta \frac{\partial u^{n}}{\partial \mathbf{n}}-C_{3}\left(u^{n}\right)^{2}+\beta C_{4} u^{n}$,

on $\Gamma^{\delta}$, for the first Stokes-like subproblem

$$
C_{1} \frac{u^{n+1-\theta}-u^{n+\theta}}{(1-2 \theta) \Delta t}+\beta \frac{\partial u^{n+1-\theta}}{\partial \mathbf{n}}+C_{3} u^{*} \cdot u^{n+1-\theta}-\beta C_{4} u^{n+1-\theta}=-\alpha \frac{\partial u^{n+\theta}}{\partial \mathbf{n}}-C_{2} \frac{\partial p^{n+\theta}}{\partial \mathbf{s}}+\alpha C_{4} u^{n+\theta},
$$

where $u^{*}$ denotes the first component of $\mathbf{u}^{*}$, on $\Gamma^{\delta}$, for the advection-diffusion-reaction subproblem, and

$$
C_{1} \frac{u^{n+1}-u^{n+1-\theta}}{\theta \Delta t}+\alpha \frac{\partial u^{n+1}}{\partial \mathbf{n}}+C_{2} \frac{\partial p^{n+1}}{\partial \mathbf{s}}-\alpha C_{4} u^{n+1}=-\beta \frac{\partial u^{n+1-\theta}}{\partial \mathbf{n}}-C_{3} u^{*} \cdot u^{n+1-\theta}+\beta C_{4} u^{n+1-\theta}
$$

for the second Stokes-like subproblem. This implementation has also being proved to be consistent with the wall law (see $[6])$. 

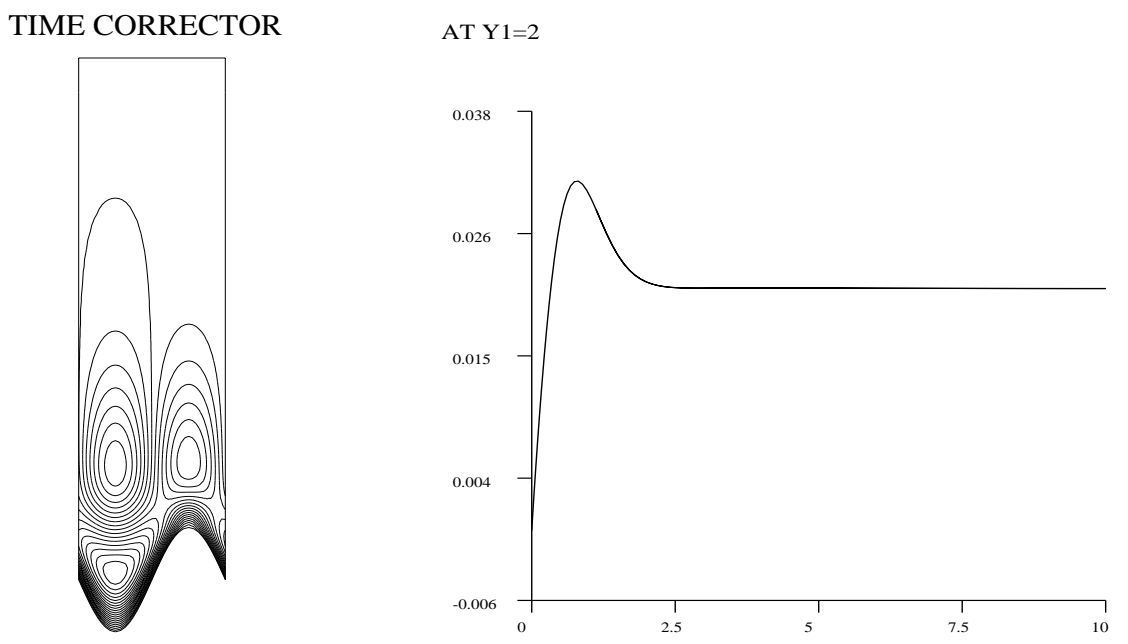

FIGURE 3. Isovalues and vertical cross section of the tangential velocity.

Finally, we remark that the spatial discretization is performed within stable $P_{2} / P_{1}$ elements $($ see $[8,14])$ for the Stokes-like subproblems, and a stabilized finite element method presented in [13] for the advection-diffusionreaction subproblem.

\section{NumERICAL EXPERIMENTS}

In this section we report three different sets of numerical experiments to validate our wall laws. The direct computation of (2.2) is done with high accuracy, with a large number of elements close to the rough wall. We investigate the first order (4.14) and second order (4.15) wall laws which allows to use a coarser mesh.

\subsection{Rough channel with a steady state limit}

The domain is the channel $[0,1] \times[0,0.5]$ with the bottom boundary divided in two parts. First, a flat plate $[0,0.18]$, followed by a rough boundary [0.18, 0.98] constituted of 20 periodic sinusoidal roughness elements of period 0.04 and amplitude 0.01. A no-slip condition is imposed in the bottom and top boundaries, while a parabolic profile is imposed on inflow boundary and a free exit is imposed on exit. For this case, we choose the magnitude of the viscosity to be $10^{-2}$, with $\mu=1$ and $\varepsilon=0.01$. The direct computation is performed with 11500 elements, and with a time step $\Delta t=0.002$.

Before employing the wall laws, we must solve the cell Stokes problems (3.7), (4.4), (4.5) and (4.6). Since the solution decays fast to a constant as $y_{2}$ grows, the cell is truncated in the $y_{2}$-direction where a homogeneous Neumann condition is imposed for the first component and a homogeneous Dirichlet condition for the second one. This decay of the solutions allows to use coarser finite element meshes. We only explicit the new cell problem (4.6), the others have been already reported in [3,21]. The horizontal size is 4 and it is truncated at $y_{2}=10$. The calculation has been made using 2500 isoparametric stabilized $Q_{1} / Q_{1}$ elements. Figure 3 depicts the contour lines of the fourth corrector $\chi^{4}$, where we can observe the fast convergence to a constant.

The values of the computed constants for the roughness element are

$$
\overline{\chi_{1}^{1}}=0.74723, \quad \overline{\chi_{1}^{2}}=-7.5 \times 10^{-4}, \quad \overline{\chi_{1}^{3}}=-0.313, \quad \overline{\chi_{1}^{4}}=0.0219 .
$$

For the first and second order effective boundary value problems, the wall laws are imposed on the line $x_{2}=$ $\delta \varepsilon=0.01$. The number of elements used is 2500 . 


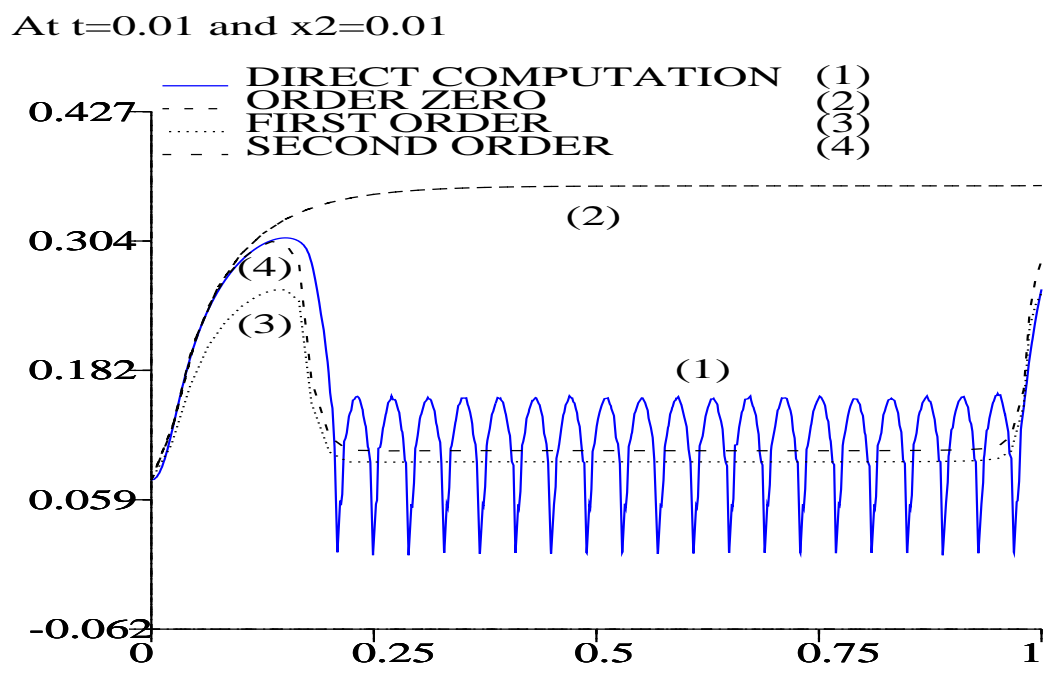

Figure 4. Cross section of the horizontal velocity.

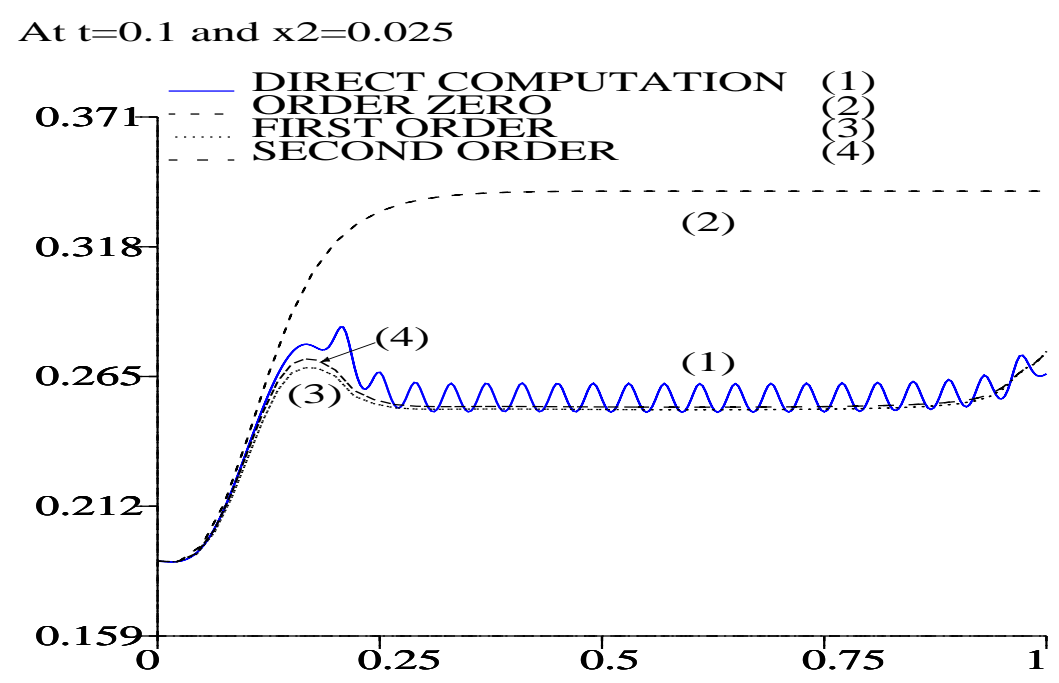

Figure 5. Cross section of the horizontal velocity.

In Figures 4 and 5 we show an horizontal cross section of the tangential velocity at $t=0.01$ and $t=0.1$, respectively. We can observe the improvement obtained by the use of high order approximations, even if, at this stage, the improvement given by the second order is not very clear, due to the fact that, since roughness are small, first order wall law gives an accurate description of the mean flow. Moreover, we can see that, for this example, the improvement provided by the second order is restricted to an interval of time in the beginning of the calculation. This shall not be the case for the subsequent examples. 


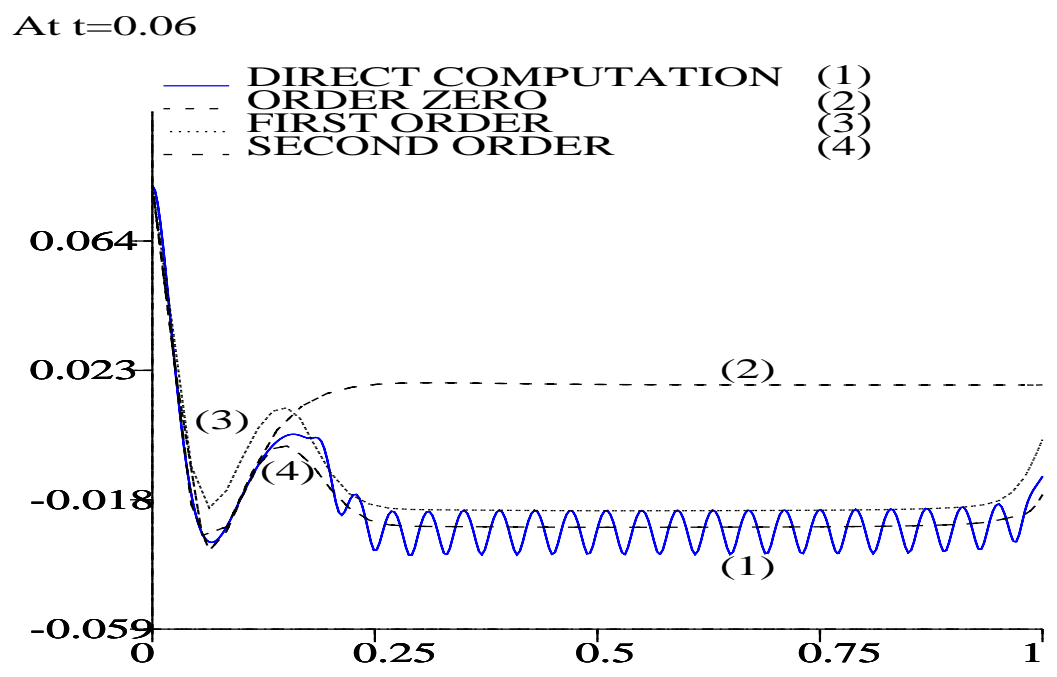

Figure 6. Cross section of the horizontal velocity.

\subsection{Unsteady rough channel}

For this case, the viscosity, geometry, meshes and time step are the same than for the previous one, but now, instead of imposing a fixed parabolic profile at entry, we impose the following oscillating function at entry:

$$
\begin{aligned}
& u_{1}\left(0, x_{2}, t\right)=16 \times x_{2} \times\left(0.5-x_{2}\right) \times(1+0.5 \times \sin (2 \pi t / 0.1)), \\
& u_{2}\left(0, x_{2}, t\right)=0
\end{aligned}
$$

To validate our wall laws, we perform horizontal cross sections of the horizontal velocity at different times. In Figures $6-8$, cross sections are presented at $t=0.06,0.08,0.1$, and at the horizontal line $x_{2}=0.015$. We may observe that the second order wall law captures the unsteadiness of the flow, while the first order wall law fails to follow the changes. In particular, in Figure 6 we see that above the roughness elements there is a recirculation zone that only the second order wall law captures completely.

\subsection{Unsteady channel with two types of roughness}

For this case we consider the channel $[0,0.9] \times[0,0.5]$ in which the bottom boundary is constituted of a flat plate on the interval $[0,0.1]$, followed by a rough boundary in which the interval $[0.1,0.7]$ is made up of 12 periodic roughness of semi-circunferencial form with radius equal to 0.015 , separated by straight lines in such a way that the whole roughness element is of an amplitude of 0.05 , and the interval [0.7,0.9] has 4 non symmetric roughness of height 0.01 and amplitude 0.05 (see Fig. 9 below). In the top and bottom boundaries a no-slip condition is considered, we take the periodic in time parabolic profile from (6.1) at inflow, and impose a free exit on outflow. The viscosity is taken as $\nu=0.003$, with $\mu=0.2$ and $\varepsilon=0.015$. Both first and second order wall laws are imposed at the line $x_{2}=\delta \varepsilon=0.015$. The direct computation has been made using $16500 P_{2} / P_{1}$ elements, specially concentrated in the region close to the rough boundary, while the computations using wall laws have been performed using 3000 elements.

The cell problems have been solved using 2900 stabilized $Q_{1} / Q_{1}$ elements. In Figures 10-13 we report the isovalues and a vertical cross section of the tangential velocity for the first order cell problem (3.7) and for the new corrector problem (4.6), for both geometries, and we observe the fast convergence to a constant in the cell 


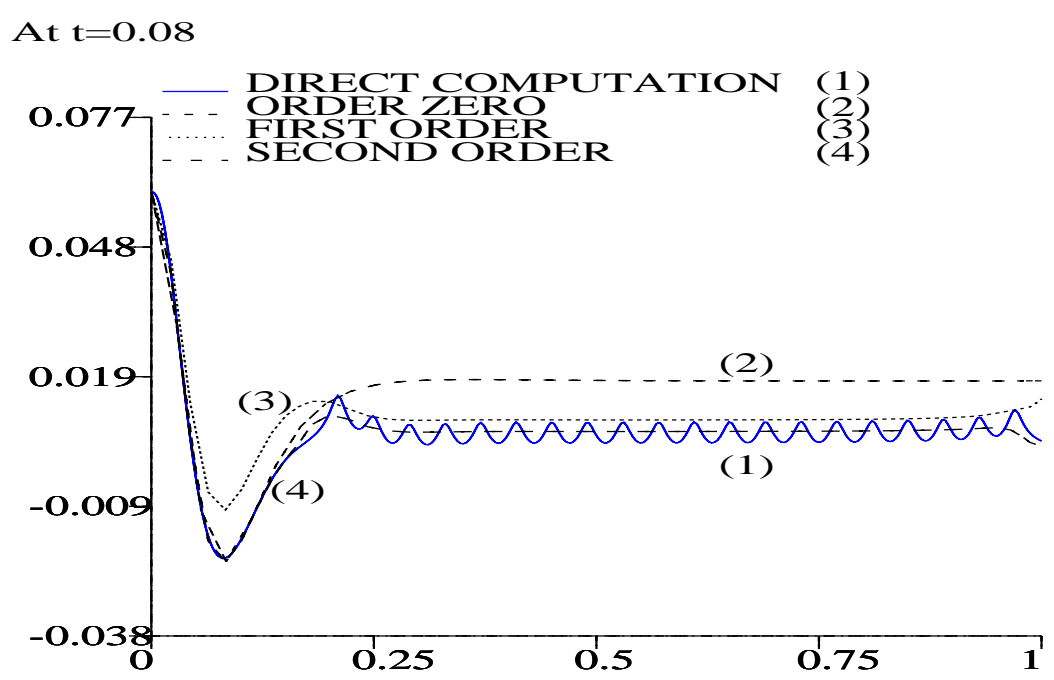

FiguRE 7. Cross section of the horizontal velocity.

At $t=0.1$

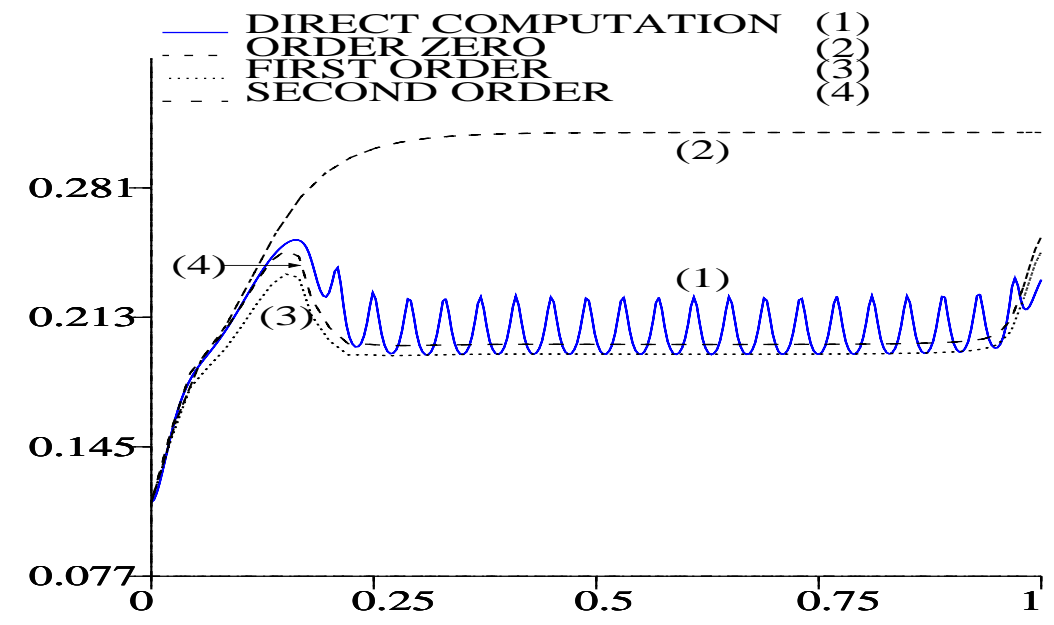

Figure 8. Cross section of the horizontal velocity.

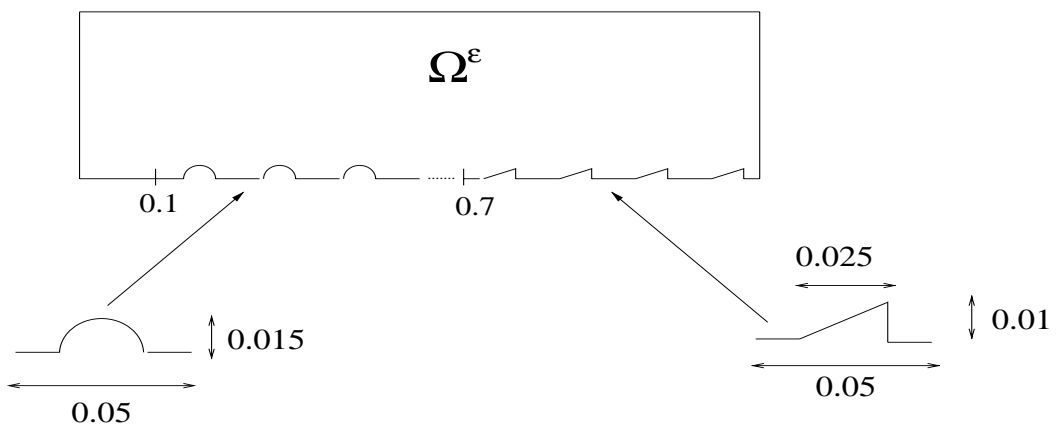

Figure 9. Geometry of the problem. 
FIRST CORECTOR : FIRST CELL

$$
\text { At } \mathrm{Y} 1=1.6
$$
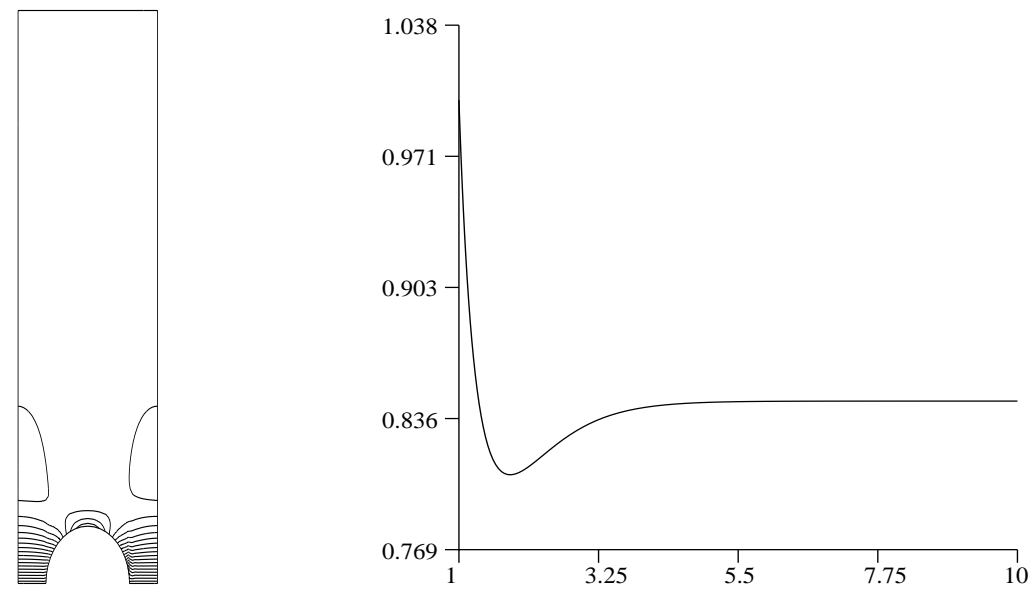

FIGURE 10. Isovalues and vertical cross section of the tangential velocity.

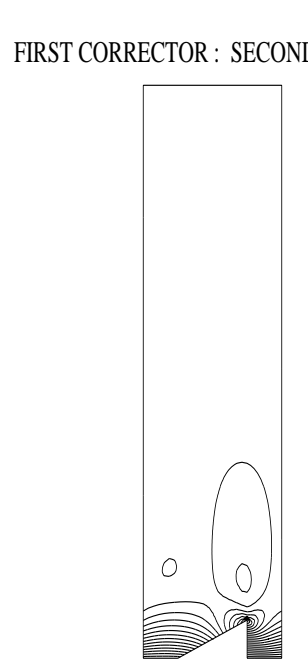

$$
\text { At } \mathrm{Y} 1=1.6
$$

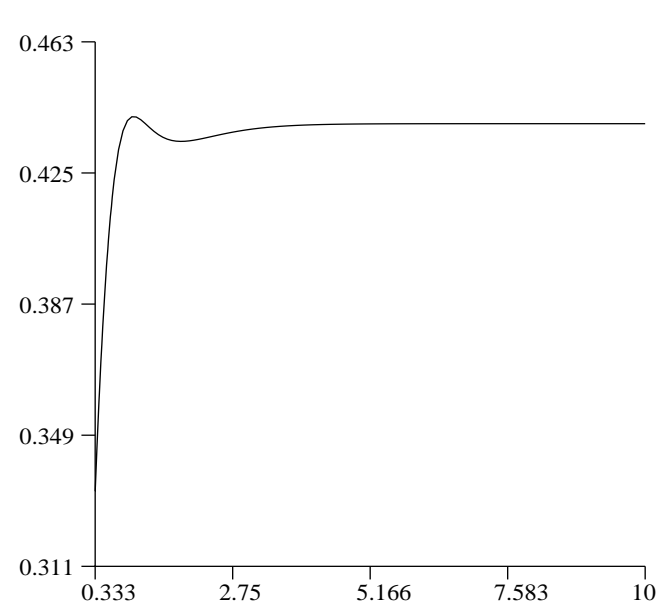

FIGURE 11. Isovalues and vertical cross section of the tangential velocity.

solutions. The obtained values for the two kind of roughness are

$$
\overline{\chi_{1}^{1}}=0.84535, \quad \overline{\chi_{1}^{2}}=-1.31 \times 10^{-3}, \quad \overline{\chi_{1}^{3}}=-0.3673, \quad \overline{\chi_{1}^{4}}=0.052,
$$

for the semi-circunferencial roughness, and

$$
\overline{\chi_{1}^{1}}=0.43968, \quad \overline{\chi_{1}^{2}}=2.5 \times 10^{-4}, \quad \overline{\chi_{1}^{3}}=-0.1171, \quad \overline{\chi_{1}^{4}}=0.053,
$$

for the non symmetric one.

We first present the isovalues of the tangential velocity in Figure 14, where we see the improvement provided by the use of higher order wall laws. 


\section{TIME CORRECTOR : FIRST CELL}

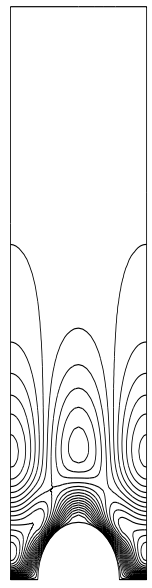

At $\mathrm{Y} 1=1.6$

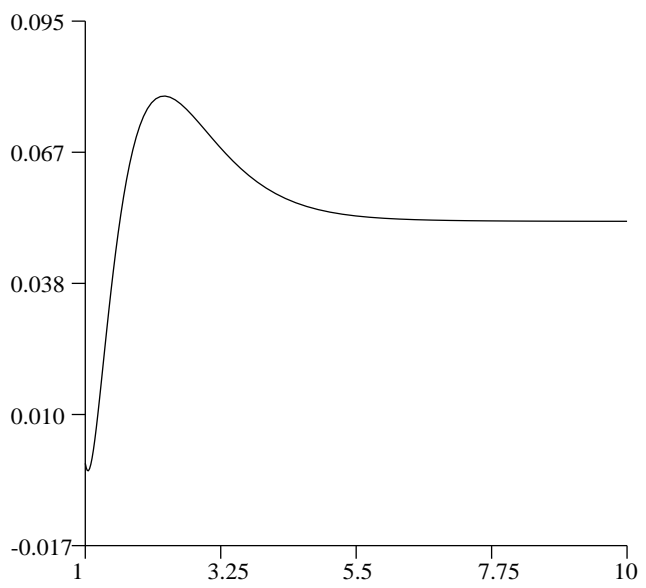

FiguRE 12. Isovalues and vertical cross section of the tangential velocity.

TIME CORRECTOR : SECOND CELL

$$
\text { At } \mathrm{Y} 1=1.6
$$
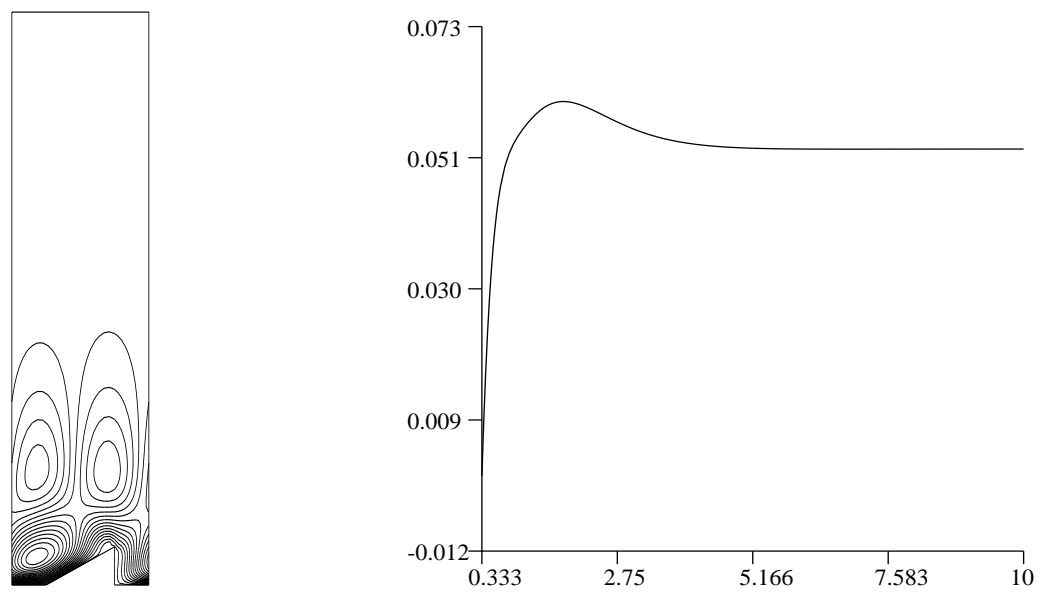

FIGURE 13. Isovalues and vertical cross section of the tangential velocity.

In Figures 15-17 we plot horizontal cross sections of the horizontal velocity at different heights and at $t=0.04,0.07,0.08$. We observe the improvement provided by the second order wall law, which is specially notorious in the transition zones between different kind of roughness, which leads to a notorious difference between both approximations in the zone above the second type of roughness. There is also a difference from the point of view of the following of the changes in time of the flow, as was pointed out in last section. We stress the fact that second order wall law provides a very good approximation of the mean flow. We remark once again the presence of a recirculation zone above the roughness elements ( $c f$. Fig. 17) that is captured only by second order wall law. Finally, in Figure 18 we present the evolution of tangential velocity at the point 
DIRECT COMPUTATION

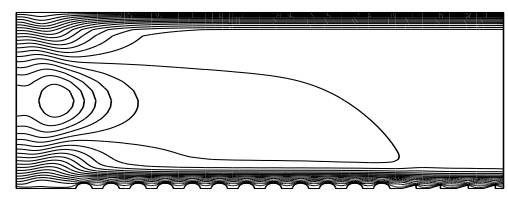

FIRST ORDER

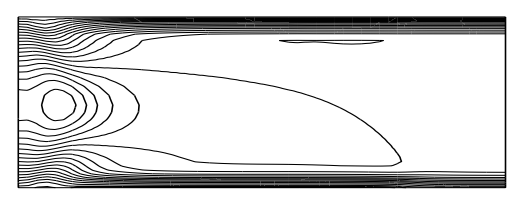

ORDER ZERO

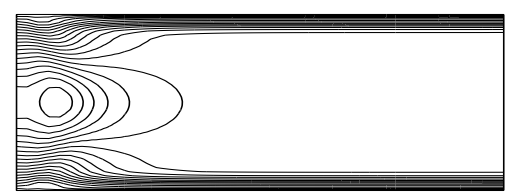

SECOND ORDER

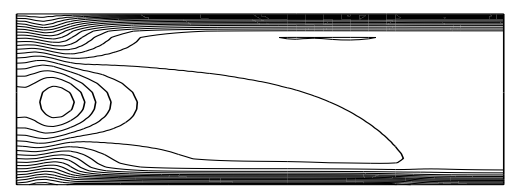

FIGURE 14. Isovalues and vertical cross section of the tangential velocity.

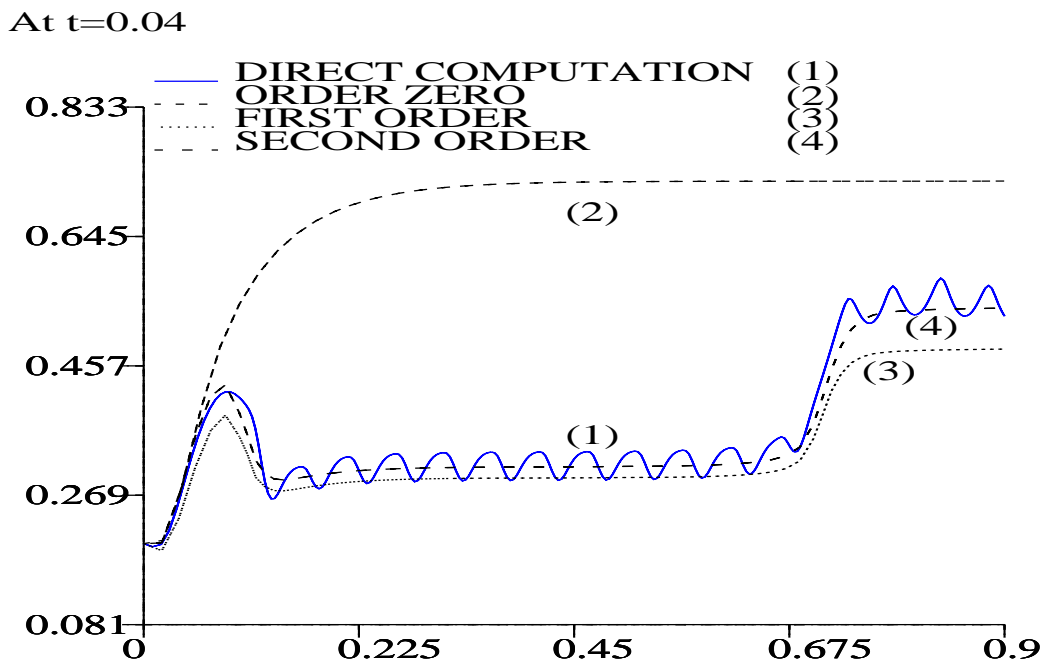

FiguRE 15. Cross section of the horizontal velocity.

$\left(x_{1}, x_{2}\right)=(0.9,0.02)$ along the first period of in time. We can observe that higher order wall laws improve drastically the approximation, and that second order wall law is more efficient. The time derivative term is the responsible for this behavior. Indeed, if we retire this term from the formulation and use the same steady wall law from [3], the approximation degradates since in that case the approximation is no longer second order accurate.

\section{Conclusion}

In contrast to the first order unsteady wall law which is the same as adopted for steady flow, second order wall law takes explicitly into account the unsteady nature of the flow through a new term in the law and a new corrector problem, which play an important role in the numerical results as it has been shown in last section, specially for the case in which the flow was unsteady. We stress the fact that the derivation of the wall 


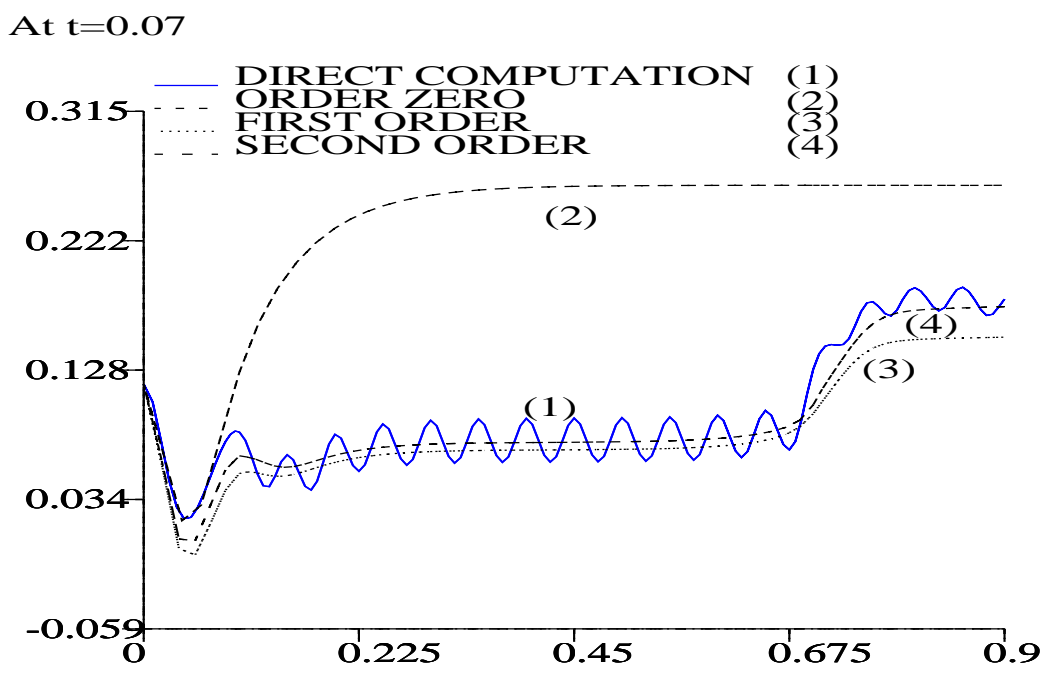

FiguRE 16. Cross section of the horizontal velocity.

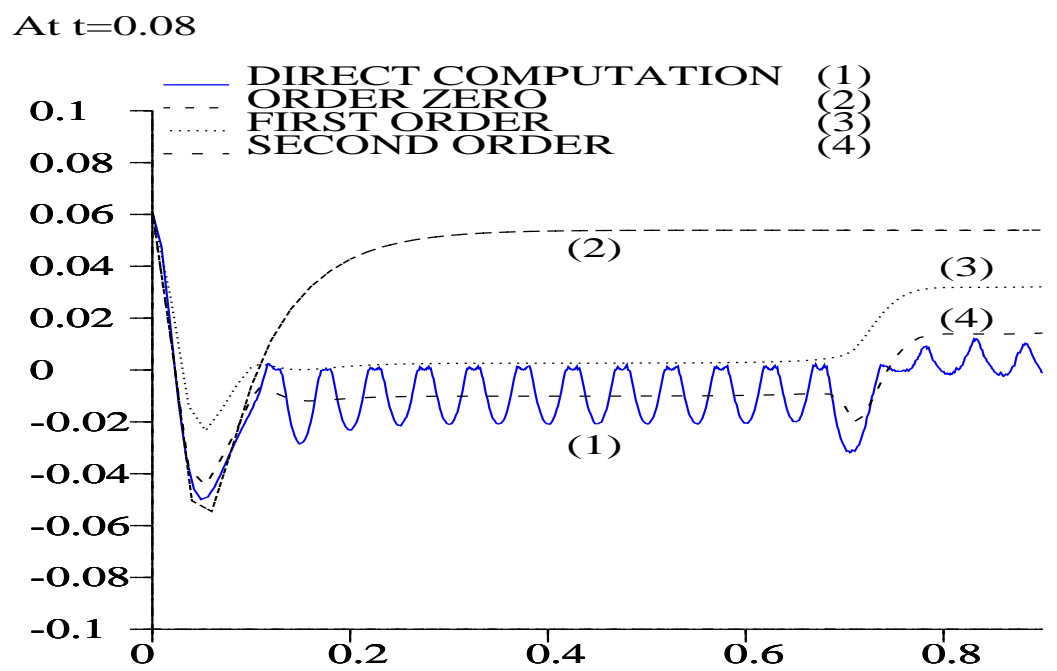

FIGURE 17. Cross section of the horizontal velocity.

laws lies in the laminar flow setting. One possibility to construct wall laws when the flow is almost turbulent is to consider a higher order asymptotic expansion. In this case, the next laws may present non-zero second component of velocity which improves the numerical results, as in has been shown in [21] for the steady case. Another possibility to treat higher Reynolds number flow is to change the viscosity scale assumption $(\nu=\mu \varepsilon)$ and to perform a different asymptotic expansion. These matters, as well as theoretical issues as existence of solutions and energy conservation will be the subject of future research. 


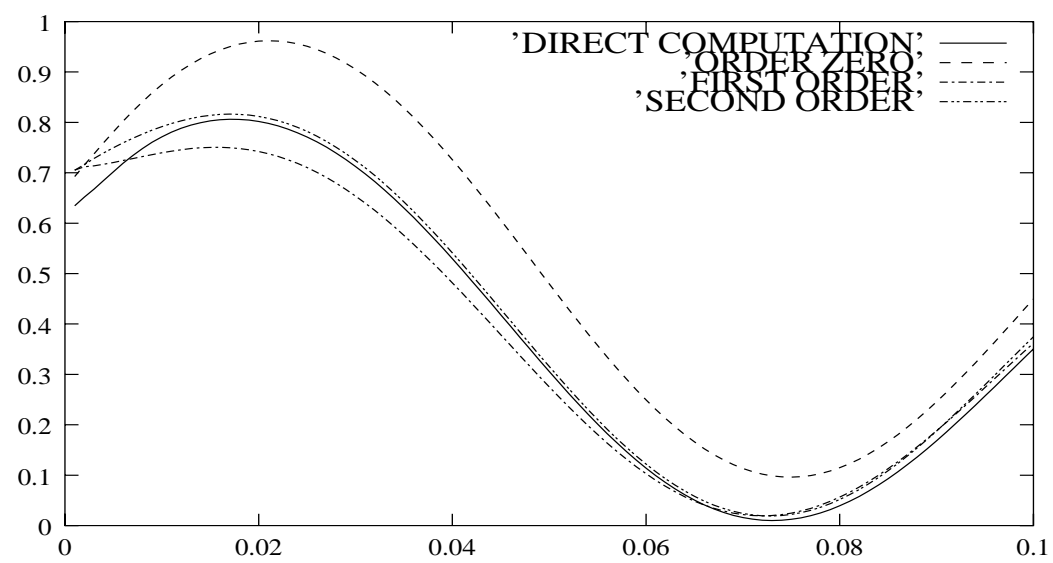

FIGURE 18. Horizontal velocity.

\section{Appendix A: Existence And uniqueness Results for the CELl PROBlems}

We now give an existence result which applies to both cell problems (4.6) and (4.4).

Theorem A.1. Let $\mathbf{g}:=\left(g^{1}, g^{2}\right) \in \mathcal{S}_{\text {per }}(Y)^{2} \cap H^{-1}(Y)^{2}$ be a function such that, for $y_{2}>H$ (with $H>0$ sufficiently large), $\mathbf{g}$ admits the following Fourier expansion

$$
g^{\gamma}(\mathbf{y})=\sum_{k=-\infty}^{+\infty} p_{k}^{\gamma}\left(y_{2}-H\right) \mathrm{e}^{-|k|\left(y_{2}-H\right)} \mathrm{e}^{i k y_{1}} \quad \gamma=1,2
$$

where, for each $k \in \mathbb{Z}, p_{k}^{1}(t)=\sum_{l=0}^{p} \alpha_{l}^{k} t^{l}$ and $p_{k}^{2}(t)=\sum_{l=0}^{p} \beta_{l}^{k} t^{l}$ are polynomes of degree $p$, and $p_{0}^{1}=p_{0}^{2}=0$. Then, there exists a unique pair of functions $(\chi, \pi)$ and a unique vector $\bar{\chi} \in \mathbb{R}^{2}$ such that $\chi-\bar{\chi} \in H_{\mathrm{per}}^{1}(Y)^{2} \cap$ $\mathcal{S}_{\text {per }}(Y)^{2}, \pi \in L_{\text {per }}^{2}(Y) \cap \mathcal{S}_{\text {per }}(Y)$, weak solution of

$$
\begin{aligned}
-\mu \Delta_{\mathbf{y}} \chi+\nabla_{\mathbf{y}} \pi & =\mathbf{g} \quad \text { in } Y, \\
\nabla_{\mathbf{y}} \cdot \chi & =0 \quad \text { in } Y, \\
\chi & =\mathbf{0} \quad \text { on } \partial Y_{3}, \\
\chi-\bar{\chi} & \in \mathcal{S}_{\text {per }}(Y)^{2}, \pi \in \mathcal{S}_{\text {per }}(Y) .
\end{aligned}
$$

Proof. The proof consists in studying an equivalent boundary value problem obtained by truncating the cell $Y$ in the $y_{2}$ direction. For the sake of simplicity we suppose that $\mu=1$, and we denote $\bar{\chi}$ by $\langle\chi\rangle$. Let $Y_{H}:=Y \cap\left\{y_{2} \leq H\right\}$ and $\Sigma_{H}:=\partial Y \cap\left\{y_{2}=H\right\}$. Assume that problem (A.1) has one solution; then, for $y_{2} \geq H$, these functions can be expressed by means of the following Fourier expansion in the $y_{1}$ variable:

$$
\chi_{1}(\mathbf{y})=\sum_{k=-\infty}^{+\infty} \chi_{1, k}\left(y_{2}\right) \mathrm{e}^{i k y_{1}}, \quad \chi_{2}(\mathbf{y})=\sum_{k=-\infty}^{+\infty} \chi_{2, k}\left(y_{2}\right) \mathrm{e}^{i k y_{1}}, \quad \pi(\mathbf{y})=\sum_{k=-\infty}^{+\infty} \pi_{k}\left(y_{2}\right) \mathrm{e}^{i k y_{1}}
$$

Hence, from the Stokes equations we see that the Fourier coefficients must satisfy the following system outside $Y_{H}$

$$
\begin{aligned}
-\chi_{1, k}^{\prime \prime}+k^{2} \chi_{1, k}+i k \pi_{k} & =p_{k}^{1}\left(y_{2}-H\right) \mathrm{e}^{-|k|\left(y_{2}-H\right)} \\
-\chi_{2, k}^{\prime \prime}+k^{2} \chi_{2, k}+\pi_{k}^{\prime} & =p_{k}^{2}\left(y_{2}-H\right) \mathrm{e}^{-|k|\left(y_{2}-H\right)} \\
i k \chi_{1, k}+\chi_{2, k}^{\prime} & =0
\end{aligned}
$$


together with the boundary conditions

- $\chi_{i, k}(H)$ given, for $i=1,2\left(H^{1}\right.$ continuity of the velocity on $\left.\Sigma_{H}\right)$,

- $\lim _{y_{2} \rightarrow \infty} \chi_{i, k}\left(y_{2}\right)=0, i=1,2$, for $k \neq 0$,

- $\lim _{y_{2} \rightarrow \infty} \chi_{i, 0}\left(y_{2}\right)=$ constant, $i=1,2$,

- $\lim _{y_{2} \rightarrow \infty} \pi_{k}\left(y_{2}\right)=0$ for $k \in \mathbb{Z}$,

the limit being reached exponentially fast.

The solution of this system may be calculated for $k \neq 0$ (see [6] for the details in the case of Problem (4.6)). Indeed, we can check that the solution of (A.3) is given by

$$
\begin{aligned}
\bullet \chi_{1, k}\left(y_{2}\right)= & {\left[\chi_{1, k}(H)+\left\{k\left(-\operatorname{sgn}(k) \chi_{1, k}(H)-i \chi_{2, k}(H)\right)\right.\right.} \\
& \left.+\sum_{l=0}^{p} \frac{(\operatorname{sgn}(k))^{l} l !}{(2 k)^{l+1}}\left(\frac{-\operatorname{sgn}(k)(l-1) \alpha_{l}^{k}+(l+1) i \beta_{l}^{k}}{2}\right)\right\}\left(y_{2}-H\right) \\
& +\left\{\frac{1}{8}\left(-\alpha_{0}^{k}-\operatorname{sgn}(k) i \beta_{0}^{k}\right)+\sum_{l=1}^{p} \frac{(\operatorname{sgn}(k))^{l-1} l !}{4(2 k)^{l}}\left(\frac{-\operatorname{sgn}(k)(l-3) \alpha_{l}^{k}+(l-1) i \beta_{l}^{k}}{2}\right)\right\}\left(y_{2}-H\right)^{2} \\
& +\left\{\frac{1}{24}\left(-\alpha_{1}^{k}-\operatorname{sgn}(k) i \beta_{1}^{k}\right)+\sum_{l=2}^{p} \frac{(\operatorname{sgn}(k))^{l} l !}{12(2 k)^{l-1}}\left(\frac{-\operatorname{sgn}(k)(l-4) \alpha_{l}^{k}+(l-2) i \beta_{l}^{k}}{2}\right)\right\}\left(y_{2}-H\right)^{3} \\
& +\sum_{l=2}^{p} \sum_{j=4}^{l+1} \frac{(\operatorname{sgn}(k))^{l+1-j} l !}{2 j !(2 k)^{l+2-j}}\left(\frac{-\operatorname{sgn}(k)(l-(j+1)) \alpha_{l}^{k}+(l-(j-1)) i \beta_{l}^{k}}{2}\right)\left(y_{2}-H\right)^{j} \\
& \left.+\sum_{l=2}^{p} \frac{1}{4(l+1)(l+2)}\left(y_{2}-H\right)^{l+2}\right] \mathrm{e}^{-|k|\left(y_{2}-H\right)},
\end{aligned}
$$

- $\chi_{2, k}\left(y_{2}\right)=\mathrm{e}^{-|k|\left(y_{2}-H\right)}\left[\chi_{2, k}(H)+k\left(-i \chi_{1, k}(H)+\operatorname{sgn}(k) \chi_{2, k}(H)\right)\left(y_{2}-H\right)\right.$

$$
\begin{aligned}
& +\sum_{l=0}^{p} \frac{(\operatorname{sgn}(k))^{l} l !}{4(2 k)^{l}}\left(\frac{\operatorname{sgn}(k)(l-1) i \alpha_{l}^{k}+(l+1) \beta_{l}^{k}}{2}\right)\left(y_{2}-H\right)^{2} \\
& +\sum_{l=1}^{p} \frac{(\operatorname{sgn}(k))^{l-1} l !}{12(2 k)^{l-1}}\left(\frac{\operatorname{sgn}(k)(l-2) i \alpha_{l}^{k}+l \beta_{l}^{k}}{2}\right)\left(y_{2}-H\right)^{3} \\
& \left.+\sum_{l=2}^{p} \sum_{j=4}^{l+2} \frac{(\operatorname{sgn}(k))^{l-j} l !}{2 j !(2 k)^{l+2-j}}\left(\frac{\operatorname{sgn}(k)(l-(j-1)) i \alpha_{l}^{k}+(l-(j-3)) \beta_{l}^{k}}{2}\right)\left(y_{2}-H\right)^{j}\right],
\end{aligned}
$$




$$
\begin{aligned}
\bullet \pi_{k}\left(y_{2}\right)= & {\left[2 k\left(-i \chi_{1, k}(H)+\operatorname{sgn}(k) \chi_{2, k}(H)\right)\right.} \\
& +\sum_{l=0}^{p} \frac{(\operatorname{sgn}(k))^{l} l !}{(2 k)^{l+1}} \frac{-(l+1) i \alpha_{l}^{k}-\operatorname{sgn}(k)(l+3) \beta_{l}^{k}}{2} \\
& +\left(\frac{1}{2}\left(-\operatorname{sgn}(k) i \alpha_{0}^{k}+\beta_{0}^{k}\right)+\sum_{l=1}^{p} \frac{(\operatorname{sgn}(k))^{l-1} l !}{2(2 k)^{l}}\left(-i \alpha_{l}^{k}-\operatorname{sgn}(k) \beta_{l}^{k}\right)\right)\left(y_{2}-H\right) \\
& +\left(\frac{1}{4}\left(-\operatorname{sgn}(k) i \alpha_{1}^{k}+\beta_{1}^{k}\right)+\sum_{l=2}^{p} \frac{(\operatorname{sgn}(k))^{l} l !}{4(2 k)^{l-1}}\left(-i \alpha_{l}^{k}-\operatorname{sgn}(k) \beta_{l}^{k}\right)\right)\left(y_{2}-H\right)^{2} \\
& +\sum_{l=2}^{p} \sum_{j=3}^{l} \frac{(\operatorname{sgn}(k))^{l-j} l !}{2 j !(2 k)^{l+1-j}}\left(-i \alpha_{l}^{k}-\operatorname{sgn}(k) \beta_{l}^{k}\right)\left(y_{2}-H\right)^{j} \\
& \left.+\sum_{l=2}^{p} \frac{1}{2(l+1)}\left(-\operatorname{sgn}(k) i \alpha_{l}^{k}+\beta_{l}^{k}\right)\left(y_{2}-H\right)^{l+1}\right] \mathrm{e}^{-|k|\left(y_{2}-H\right)} .
\end{aligned}
$$

In other words, the coefficients $\chi_{i, k}, i=1,2$ and $\pi_{k}$ are polynomials of degree $p+2$ and $p+1$ in $\left(y_{2}-H\right)$, respectively, within the multiplicative coefficient $\mathrm{e}^{-|k|\left(y_{2}-H\right)}$.

For $k=0, \chi_{1,0}$ and $\chi_{2,0}$ do not depend on $y_{2}$, and hence the constant $\langle\chi\rangle$ is given by $\langle\chi\rangle=\left(\chi_{1,0}, \chi_{2,0}\right)$. To see this, we write the system for $k=0$, yielding

$$
\begin{array}{rlrl}
\chi_{1,0}^{\prime \prime} & =p_{0}^{1}=0 & & \text { (by assumption) }, \\
\pi_{0}^{\prime} & =p_{0}^{2}=0 & & \text { (by assumption) }, \\
\chi_{2,0}^{\prime} & =0, &
\end{array}
$$

and from the boundary conditions at infinity we get $\pi_{0}=0, \chi_{1,0}=\left\langle\chi_{1}\right\rangle$ and $\chi_{2,0}=\left\langle\chi_{2}\right\rangle$.

Now, to build an equivalent boundary condition on $\Sigma_{H}$ we calculate:

$$
\begin{aligned}
-\frac{\partial \chi_{1, k}}{\partial y_{2}} & =-k\left(-2 \operatorname{sgn}(k) \chi_{1, k}(H)-i \chi_{2, k}(H)\right)-\sum_{l=0}^{p} \frac{(\operatorname{sgn}(k))^{l} l !}{(2 k)^{l+1}}\left(\frac{-\operatorname{sgn}(k)(l-1) \alpha_{l}^{k}+(l+1) i \beta_{l}^{k}}{2}\right), \\
-\frac{\partial \chi_{2, k}}{\partial y_{2}}+\pi & =k\left(-i \chi_{1, k}(H)+2 \operatorname{sgn}(k) \chi_{2, k}(H)\right)+\sum_{l=0}^{p} \frac{(\operatorname{sgn}(k))^{l} l !}{(2 k)^{l+1}}\left(\frac{-(l+1) i \alpha_{l}^{k}-\operatorname{sgn}(k)(l+3) \beta_{l}^{k}}{2}\right) .
\end{aligned}
$$

Hence, after solving the equation outside $Y_{H}$ and substitution, we obtain the following equivalent boundary condition

$$
-\frac{\partial \chi}{\partial y_{2}}+\pi \mathbf{e}_{2}=\mathcal{T} \chi+\mathcal{H} \mathbf{g} \quad \text { on } \Sigma_{H}
$$

where $\mathcal{T} \chi$ and $\mathcal{H}$ g are given by

$$
\mathcal{T} \chi=\left(\begin{array}{c}
\sum_{k=-\infty}^{+\infty} k\left(2 \operatorname{sgn}(k) \chi_{1, k}(H)+i \chi_{2, k}(H)\right) \mathrm{e}^{i k y_{1}} \\
\sum_{k=-\infty}^{+\infty} k\left(-i \chi_{1, k}(H)+2 \operatorname{sgn}(k) \chi_{2, k}(H)\right) \mathrm{e}^{i k y_{1}}
\end{array}\right)
$$


and

$$
\mathcal{H} \mathbf{g}=\left(\begin{array}{c}
\sum_{k \neq 0} \sum_{l=0}^{p} \frac{(\operatorname{sgn}(k))^{l} l !}{(2 k)^{l+1}} \frac{\operatorname{sgn}(k)(l-1) \alpha_{l}^{k}-(l+1) i \beta_{l}^{k}}{2} \mathrm{e}^{i k y_{1}} \\
\sum_{k \neq 0} \sum_{l=0}^{p} \frac{(\operatorname{sgn}(k))^{l} l !}{(2 k)^{l+1}} \frac{-(l+1) i \alpha_{l}^{k}-\operatorname{sgn}(k)(l+3) \beta_{l}^{k}}{2} \mathrm{e}^{i k y_{1}}
\end{array}\right)
$$

The proof is then reduced to show that there exists a unique solution to the problem: Find $(\chi, \pi) \in\left(H_{\mathrm{per}}^{1}\left(Y_{H}\right)\right)^{2} \times$ $L_{\mathrm{per}}^{2}\left(Y_{H}\right)$ such that

$$
\begin{aligned}
-\Delta_{\mathbf{y} \chi} \chi \nabla_{\mathbf{y}} \pi & =\mathbf{g} & & \text { in } Y_{H}, \\
\nabla_{\mathbf{y}} \cdot \chi & =0 & & \text { in } Y_{H}, \\
\chi & =\mathbf{0} & & \text { on } \partial Y_{3}, \\
-\frac{\partial \chi}{\partial y_{2}}+\pi & =\mathcal{T} \chi+\mathcal{H} \mathbf{g} & & \text { on } \Sigma_{H},
\end{aligned}
$$

and then to extend it to the whole cell $Y$ via (A.2). Let $V$ be the space defined by

$$
V:=\left\{\chi \in\left(H_{\mathrm{per}}^{1}\left(Y_{H}\right)\right)^{2} / \nabla_{\mathbf{y}} \cdot \chi=0 \text { in } Y_{H}\right\}
$$

and

$$
V_{0}:=\left\{\chi \in V / \chi=0 \text { on } \partial Y_{3}\right\}
$$

The variational formulation of (A.7) reads: Find $\chi \in V_{0}$ such that

$$
\int_{Y_{H}} \nabla \chi: \nabla \bar{\eta}+\langle\mathcal{T} \chi, \bar{\eta}\rangle=-\int_{Y_{H}} \mathbf{g} \cdot \bar{\eta}-\langle\mathcal{H} \mathbf{g}, \bar{\eta}\rangle \quad \forall \eta \in V_{0}
$$

First, we note that the right hand side of this formulation is well defined since in $\mathcal{H} \mathbf{g}$ there is a division by at least one power of $k$, and since $\mathbf{g}$ was originally $H^{-1}$ in $y_{1}$, after division by $k, \mathcal{H} \mathbf{g}$ is $L^{2}$ in $y_{1}$. Now, to prove our result we have to show that the sesquilinear form in the left hand side of (A.8) is coercive in $V_{0}$, and then apply Lax-Milgram Lemma. The positivity comes from Poincare's inequality, and the fact that $\mathcal{T}$ is positive semi-definite. In fact, using the orthogonality of $\left\{\mathrm{e}^{i k y_{1}}\right\}_{k=-\infty}^{+\infty}$ in $L^{2}\left(\Sigma_{H}\right)$ it is not difficult to see that that

$$
\langle\mathcal{T} \chi, \bar{\chi}\rangle \geq \sum_{k=-\infty}^{+\infty} 4 \pi^{2}|k|\left(\left|\chi_{1, k}(H)\right|^{2}+\left|\chi_{2, k}(H)\right|^{2}\right) \geq 0
$$

We then apply Lax-Milgram Lemma to conclude that the problem has a unique solution, which can be extended to the whole cell $Y$ via (A.2). We have hence, since both problems (A.1) and (A.8) are equivalent, that (A.1) has a unique solution. To see that this solution is real, we remark that the real part of it satisfies (A.1) itself (because the right hand size of (A.1) is real), and then, by uniqueness we conclude that we have a real solution.

Acknowledgements. The authors would like to thank Professor Yves Achdou from Université Denis Diderot (Paris VII), France, for his helpful discussions and comments. 


\section{REFERENCES}

[1] Y. Achdou and O. Pironneau, Domain decomoposition and wall laws. C. R. Acad. Sci. Paris Série I Math. 320 (1995) $541-547$.

[2] Y. Achdou, O. Pironneau and F. Valentin, Etude des lois de paroi d'ordre 1 et 2 pour des domaines rugueux par décomposition de domaine. Technical Report 3326, INRIA (1997).

[3] Y. Achdou, O. Pironneau and F. Valentin, Effective boundary conditions for laminar flows over periodic rough boundaries. $J$. Comput. Phys. 147 (1998) 187-218.

[4] Y. Achdou, O. Pironneau and F. Valentin, Équations aux dérivées partielles et applications - Articles dédiés à Jacques-Louis Lions, chapter Shape control versus boundary control. Elsevier, Paris (1998) 1-18.

[5] Y. Achdou, P. Le Tallec, F. Valentin and O. Pironneau, Constructing wall laws with domain decomposition or asymptotic expansion techniques. Comput. Methods Appl. Mech. Engrg. 151 (1998) 215-232.

[6] G.R. Barrenechea, Analyse Numérique et Lois de Paroi pour des Écoulements Instationnaires sur des Parois Rugueuses. Ph.D. thesis, Université de Paris Dauphine (2001), in preparation.

[7] A. Bensoussan, J.L. Lions and G. Papanicolaou, Asymptotic analysis for periodic structures. North Holland (1983).

[8] F. Brezzi and M. Fortin, Mixed and Hybrid Finite Element Methods. Springer-Verlag (1991).

[9] A. Carrau, G. Galice and P. Le Tallec, Taking into account surface roughness in computing hypersonic rentry bodies. Applied Sciences and Engineering, R. Glowinski, Ed., Nova Science publisher (1992) 331-344.

[10] J. Cousteix, Couches Limites Laminaires. Cepadues (1989).

[11] E. Dean and R. Glowinski, On some finite element methods for the numerical solution of incompressible flow, in Incompressible Computational Fluid Dynamics, M. Gunzburger and R. Nicolaides, Eds., Cambridge University Press (1993).

[12] J. Dutton, Dynamics of atmospheric motion. Dover (1986).

[13] L.P. Franca and F. Valentin, On an improved unusual stabilized finite element method for the advective-reactive-diffusive equation. Comput. Methods Appl. Mech. Engrg. 190 (2000) 1785-1800.

[14] V. Girault and P.A. Raviart, Finite Element Methods for the Navier-Stokes Equations. Springer-Verlag (1986).

[15] R. Lewandowski, Analyse mathématique et océanographie. Masson (1997).

[16] B. Mohammadi and G. Medic, A critical evaluation of the classical $k$ - $\varepsilon$ model and wall-laws for unsteady flows over bluff bodies. Int. J. Comput. Fluid Dyn. 10 (1998) 1-11.

[17] B. Mohammadi and O. Pironneau, Unsteady separated turbulent flows computation with wall-laws and $k-\varepsilon$ model. Comput. Methods Appl. Mech. Engrg. 148 (1997) 393-405.

[18] E. Sánchez-Palencia, Un problème d'écoulement lent d'un fluide visqueux incompressible au travers d'une paroi finement perforée, in Les Méthodes de l'Homogénéisation: Théorie et Applications en Physique, D. Bergman et coll, Eds., Vol. 57 of Collection de la Direction des Études et Recherche d'Électricité de France, Eyrolles, Paris (1985) 371-400.

[19] A. Smith and D. Silvester, Implicit algorithms and their linearization for the transient incompressible Navier-Stokes equations. IMA J. Numer. Anal. 17 (1997) 527-545.

[20] R. Temam, Navier Stokes Equations. Theory and Numerical Algorithms. North-Holland, third edition (1984).

[21] F. Valentin, Nouvelles conditions aux limites équivalentes pour des interfaces rugueuses en mécanique des fluides : développement, analyse et mise en ouvre numérique. Ph.D. thesis, Université Paris 6 (1998).

[22] T. Yamaguchi, Computational mechanics simulation for clinical cardiovascular medicine, in ECCOMAS 2000, Barcelona (2000). 